\title{
Players in Mitochondrial Dynamics and Female Reproduction
}

\author{
Weiwei Zou ${ }^{1,2,3}$, Dongmei Ji ${ }^{1,4,5}$, Zhiguo Zhang ${ }^{1,4,5}$, Li Yang ${ }^{6 *}$ and Yunxia Cao ${ }^{1,2,3 *}$ \\ ${ }^{1}$ Reproductive Medicine Center, Department of Obstetrics and Gynecology, The First Affiliated Hospital of Anhui Medical \\ University, Hefei, China, ${ }^{2} \mathrm{NHC}$ Key Laboratory of Study on Abnormal Gametes and Reproductive Tract (Anhui Medical University), \\ Hefei, China, ${ }^{3}$ Key Laboratory of Population Health Across Life Cycle (Anhui Medical University), Ministry of Education of the \\ People's Republic of China, Hefei, China, ${ }^{4}$ Anhui Province Key Laboratory of Reproductive Health and Genetics, Hefei, China, \\ ${ }^{5}$ Biopreservation and Artificial Organs, Anhui Provincial Engineering Research Center, Anhui Medical University, Hefei, China, \\ ${ }^{6}$ Department of Pediatrics, Xiangya Hospital, Central South University, Changsha, China
}

OPEN ACCESS

Edited by:

Giuseppe Calamita,

University of Bari Aldo Moro, Italy

Reviewed by:

Marco G. Alves, Independent researcher,

Porto, Portugal

Marc Yeste,

University of Girona, Spain

*Correspondence:

Li Yang

yangli525@csu.edu.cn

Yunxia Cao

caoyunxia6@126.com

Specialty section: This article was submitted to

Cellular Biochemistry,

a section of the journal

Frontiers in Molecular Biosciences

Received: 07 June 2021 Accepted: 07 September 2021

Published: 11 October 2021

Citation:

Zou W, Ji D, Zhang Z, Yang $L$ and Cao Y (2021) Players in Mitochondrial Dynamics and Female Reproduction.

Front. Mol. Biosci. 8:717328

doi: 10.3389/fmolb.2021.717328
Mitochondrial dynamics (fission and fusion) are essential physiological processes for mitochondrial metabolic function, mitochondrial redistribution, and mitochondrial quality control. Various proteins are involved in regulating mitochondrial dynamics. Aberrant expression of these proteins interferes with mitochondrial dynamics and induces a range of diseases. Multiple therapeutic approaches have been developed to treat the related diseases in recent years, but their curative effects are limited. Meanwhile, the role of mitochondrial dynamics in female reproductive function has attracted progressively more attention, including oocyte development and maturation, fertilization, and embryonic development. Here, we reviewed the significance of mitochondrial dynamics, proteins involved in mitochondrial dynamics, and disorders resulting from primary mitochondrial dynamic dysfunction. We summarized the latest therapeutic approaches of hereditary mitochondrial fusion-fission abnormalities and reviewed the recent advances in female reproductive mitochondrial dynamics.

Keywords: mitochondrial dynamics, protein, diseases, therapy, reproduction

\section{INTRODUCTION}

Mitochondria, cellular organelles with bi-membrane structures, are essential for maintaining cell metabolism and producing energy. Mitochondrial dynamics is a continuous process of fission and fusion between the inner mitochondrial membranes (IMMs) and the outer mitochondrial membranes with other mitochondria (Wai and Langer, 2016). Mitochondria effectively modulate fission and fusion to exchange matrix and membrane components (Frederick and Shaw, 2007). The constant and homeostatic fission and fusion processes form a dynamic interconnection network that maintains the integrity of mitochondria and mtDNA (Suen et al., 2008). Impairments in mitochondrial dynamics are often related to cellular energic deficiency, especially in those tissues in high demand of energy, like neurons, cardiomyocytes, and muscle cells. In general, impaired oxidative phosphorylation (OXPHOS), mtDNA deficiency, and overproduction of reactive oxygen species (ROS) under pathological conditions induce mitochondrial fusion (Jendrach et al., 2008; Tondera et al., 2009).

Mitochondrial dysfunction tends to increase ROS production, which can lead to mtDNA mutations and cell damage. Mitochondrial fusion can either improve energy supply through expanded IMMs or promote the material exchange and complementation between healthy and defective mitochondria, ensuring mtDNA integrity and recovery of mitochondrial respiratory function (Liesa and Shirihai, 2013; Cai and Tammineni, 2016). The inhibition of mitochondrial 
fusion leads to mtDNA loss and declined mitochondrial respiration function (Clark-Walker and Miklos, 1975; Harvey, 2019). Mitochondrial fission leads to the removal of aberrant mitochondria while under stress and undergoing apoptosis and the assignment of mitochondria into daughter cells (Twig et al., 2008) (Chan, 2012). Inhibition of mitochondrial fission prevents repairing of disabled mitochondrial function (Benard et al., 2007; Twig et al., 2008).

Mitochondria are the most abundant organelles in oocytes and embryos. They undergo constant dynamic changes during oocyte maturation and embryonic development before implantation to support major cellular development events (Motta et al., 2000; Sathananthan and Trounson, 2000; Harvey, 2019). Human oocytes metabolize pyruvate via OXPHOS, primarily during the growth to provide energy (Harvey, 2019). Human oocytes and early embryo metabolism are characterized by low oxidative metabolism and oxygen consumption and the utilization of pyruvate, lactic acid, and amino acids to support development (Dumesic et al., 2015). The blastocyst stage showed high levels of glycolysis and oxygen consumption. It resulted in the activation of the embryonic genome and higher energy requirements for blastocyst formation (Gardner and Harvey, 2015). Fluctuating energy requirements promote constant variations in mitochondrial dynamics, which dynamically and harmoniously modulate metabolism to support the development of oocytes and embryos. The typical mitochondrial fission-fusion mechanism forms a dynamically interconnected network to preserve regular mitochondria activity. Meanwhile, it satisfies the material and energy demands to maintain oocyte maturation and preimplantation embryonic development (Frederick and Shaw, 2007).

This review aims to summarize the findings of current investigations into mitochondrial dynamics-related proteins, related diseases, targeted therapy, and vital functions of mitochondrial dynamics in oocyte and embryonic development. We found that the research on mitochondrial dynamics in female reproduction is quite limited. Therefore, further research and therapeutic methods will be discovered in the field, which may draw greater attention to mitochondrial dynamics and female reproduction.

\section{SIGNIFICANT PLAYERS OF MITOCHONDRIAL DYNAMICS}

\section{Dynamin-Related Protein 1}

The most knowable one of these series is DRP1, a mitochondrial fission GTPase (Otera and Mihara, 2011). Also known as DNM1L (Dynamin 1-like), DRP1 is a member of the dynamin family of large GTPases. DRP1 shares a similar architecture with the other dynamin superfamily members. The polypeptide chain folds backward to form a monomer with four domains (the head, neck, trunk, and foot). The head consists of a GTP-binding G domain, the trunk contains a self-organizing interface, and the neck is an essential component of the bundle signal (Reubold et al., 2015; Kalia et al., 2018). The foot is a variable domain containing approximately 100 amino acid residues that bind negative-charged lipids such as cardiolipin and phosphatidic acid (Stepanyants et al., 2015; Adachi et al., 2016). It controls the final part of mitochondrial fission, pinching off the membrane stalk between two daughter mitochondria. Without normal functioned DRP1, the tubular projections of mitochondria typically present in cells are retracted into large perinuclear aggregates (Smirnova et al., 1998). Therefore, pioneering researchers have suggested that DRP1 maintains mitochondrial morphology by distributing the yeast Dnm1 corresponding mitochondrial tubules throughout the cytoplasm. Additional studies have shown that DRP1 plays a role in programmed cell death during $C$. elegans development. DRP1 is also involved in the restructuring and opening of mitochondrial cristae during apoptosis (Wang et al., 2012). Furthermore, DRP1 induces programmed cell death via the ligation to CD47, which requires its translocation from cytosol to mitochondria (Kamerkar et al., 2018). Moreover, DRP1 is also essential for maintaining mitochondrial health. In heteroplasmic m.3243 A > G cells, silencing of DRP1 was associated with increased levels of mutant mitochondrial DNA (Malena et al., 2009). Finally, DRP1 has to be precisely modified to functions normally. IFN- $\beta$, a mitochondrial fission initiator, phosphorylates STAT5 and upregulates PGAM5, phosphorylating serine 622 of DRP1 (Tresse et al., 2021). Phosphorylated PGAM5S, a complex required during necrosis, recruits DRP1. It activates DRP1 by dephosphorylation and induces mitochondrial fragmentation and execution of necrosis (Wang et al., 2012). Conversely, DRP1 knockdown leads to elongated peroxisomes, indicating a ubiquitous divisome function of DRP1 (Kamerkar et al., 2018). DRP1 needs adaptors to anchor the mitochondrial outer membrane inside the cell. These adaptors were first identified in yeast, including Fis1, Mdv1, and Caf4. There is no homolog in metazoans of Mdv1 and Caf4, and the mammalian has their specific adaptor for DRP1. This series of proteins comprise the mitochondrial fission factor (MFF) (Gandre-Babbe and van der Bliek, 2008) and the chordate-specific mitochondrial dynamics proteins of $49 \mathrm{kDa}$ (MiD49), also known as MIEF2, and $51 \mathrm{kDa}$ (MiD51), also known as MIEF1 (Otera et al., 2016). Those adaptors can recruit DRP1 solely; ablation of their function will lead to dysregulation of mitochondrial fission.

\section{Inverted Formin 2}

INF2, also known as ER-localized inverted formin 2, is a member of the formin family, binds to the barbed ends of developing filaments, and protects them from capping. They create long actin filaments to cross-link into bundles. IFN2 interacts with the formin homology-1 and 2 (FH1 and 2) domain, gelsolin, and profilin to block the capping of the barbed end and create short filaments of actin subunits through a combination of barbed end elongation, severing, and WH2 motif-mediated depolymerization (Bindschadler and McGrath, 2004; Chhabra and Higgs, 2006; Gurel et al., 2015). INF2 was an upstream factor of DRP1. Actin filaments accumulate between mitochondria and INF2-enriched ER membranes at constriction sites. Some research studies indicate that INF2 enrichment might be the initial step of mitochondrial constriction before the accumulation of DRP1 
(Korobova et al., 2013). Subsequently, IFN stabilizes the ER-mitochondrial platforms and tethers the damaged mitochondria to the ER, separating them through fission (Tresse et al., 2021). ER-mitochondria contacts increase calcium uptake by mitochondria and mitochondrial division (Steffen and Koehler, 2018). Furthermore, an actin-nucleating protein, Spire 1C, directly links mitochondria to the actin cytoskeleton and the ER. Spire 1C cooperates with IFN2 and promotes actin assembly on the mitochondrial surface, driving one of the initial steps of mitochondrial fission (Manor et al., 2015).

\section{Mitochondrial Fission 1}

FIS1 was first discovered in yeast as a gene capable of relieving temperature-sensitive alleles of the fusion genes (James et al., 2003). Its human orthologue, also known as hFis1, is a component of a mitochondrial complex that promotes mitochondrial fission (James et al., 2003). FIS1, localized to the outer mitochondrial membrane, was first thought to be a helper factor of DRP1 during mitochondrial fission and cellular apoptosis. The C-terminus anchor of FIS1 was crucial for the localization of mitochondria. A $15-\mathrm{kDa}$ soluble domain inside this domain with two tetratricopeptide repeats (TPRs) acts as the tethering site of the mitochondrial outer membrane. The subsequent study found that FS1 recruits DRP1 from the cytosol to the fission site of mitochondria (Ihenacho et al., 2021). Coimmunoprecipitation studies suggest that FIS1 may act as a downstream factor of Mff of DRP1 recruitment and assembly at scission sites (Shen et al., 2014). Knockout of Fis1 and Mff simultaneously resulted in a more pronounced mitochondrial elongation phenotype than knockout of them independently, suggesting an independent role of the above two during mitochondrial fission (Shen et al., 2014).

The number of FS1 molecules is the limiting factor of the frequency of mitochondria fission (Yoon et al., 2003). Although FIS1 plays a significant role in mitochondria fission, overexpression of FIS1 makes no alternation on the membrane potential, $\mathrm{PH}$ value, or the calcium cation capacity of mitochondria (Frieden et al., 2004). Like DRP1, attenuation of FIS1 led to the increase in heteroplasmy of pathological m.3243 A $>\mathrm{G}$ mutation (Malena et al., 2009). In addition, FIS1, which is also colocalized with DRP1 during peroxisome fission (Kobayashi et al., 2007), may have a role in cellular apoptosis. However, studies in lower animals found controversial results compared to those in mammalian cells. The ablation of Fis1 in C. elegans did not elongate mitochondria as expected (Breckenridge et al., 2008), while the embryonic fibroblast of the $F i s 1^{-1-}$ mouse presented with elongated mitochondria. Additionally, the knockout of Fis1 in mice is embryonic lethal (Losón et al., 2013). Many studies have explored the relationship between FIS1 and other proteins that play a role in mitochondrial dynamics. A report proposed that FIS1 may act as the negative regulator of Mid51 while recruiting DRP1 (Zhao et al., 2011). Studies showed that FIS1 was a stress-specific DRP1 recruiter, while Mff was the primary recruiter of DRP1 in mammalian cells (Otera et al., 2010; Otera et al., 2016). On the other hand, Fis1 activated Caspase- 8 during cell damage
(Iwasawa et al., 2011); furthermore, Fis1 induces hierarchical macroautophagy and inhibition of syntaxin 17, indicating that Fis1 is involved in both mitophagy and apoptosis (Xian et al., 2019). A recent study claimed that Fis 1 dominated the peripheral mitochondrial fission, enabling damaged material to be shed into smaller mitochondria, ultimately directing to mitophagy (Kleele et al., 2021). Those pieces of evidence emphasize the stressreactor role of Fis1. However, the primary function of FIS1 in the cell remains fully elucidated.

\section{Mitochondrial Fission Factor}

MFF is a 342 amino acid protein containing two short repeat motifs close to the N-terminus, followed by a helical coil domain, a transmembrane domain, and a short C-terminal tail. Alternative splicing of MFF results in 19 transcript variants locating either on the cell membrane or the cytosol. Mff was first anchored via its tail to the mitochondrial outer membrane with Fis1 in drosophila cells (Gandre-Babbe and van der Bliek, 2008). Activation of adenosine monophosphate (AMP)-activated protein kinase (AMPK) resulted in rapid mitochondrial fragmentation. The substrate screening of AMPK has revealed that Mff catalyzes the guanosine triphosphatase category, which speeds up mitochondrial fission (Toyama et al., 2016). A recent study in mouse cardiomyocytes found that Mff only regulates cellular midzone fission. The fission that occurs in the midzone is unlikely to colocalize with the lysosome. They also found that fission in the peripheral area is frequently combined with lysosome recruiting, suggesting that it is responsible for mitochondria proliferation (Kleele et al., 2021). This research might be the potential explanation for why MFF and FIS1 act so distinctly.

\section{Mitochondrial Dynamics Proteins of 49 kDa and $51 \mathrm{kDa}$}

Also known as MIEF1 and MIEF2, both have nucleotidyltransferase folds with an N-terminal transmembrane anchor for integration into the mitochondrial outer membrane (Osellame et al., 2016). They share many architectural similarities. However, only MID51 can bind nucleotide diphosphates (ADP and GDP), whereas the ligand binding MID49 is still unknown. MID51 is dimeric; in contrast, MID49 is monomeric, but both share motifs interacting with DRP1 (Losón et al., 2014). Their structural differences might suggest a differential regulation and stabilization of MID51 versus MID49 (Richter et al., 2014; Losón et al., 2015). Different reports have ascribed opposing roles to these two proteins in fission and fusion. Initially, researchers found that overexpression of MID49 or MID51 caused mitochondrial fusion, elongating mitochondrial tubules projecting out of a dissociated perinuclear network (Palmer et al., 2011). Afterward, they found that the fusogenic effect was due to sequestration and inhibition of DRP1, allowing unchecked mitochondrial fusion by MFN1 and MFN2 alongside peroxisome fusion in mouse embryonic fibroblasts. They proposed that MID49 and MID51 could function in mitochondrial fission under common conditions (Palmer et al., 2013). Recent patient research 
proved this hypothesis further. The MID49 defect elevated mitochondrial fusion frequency and ragged-red fibers in patient's muscles (Bartsakoulia et al., 2018). Surprisingly, the mtDNA levels of patient fibroblasts increased, along with the mildly increased OXPHOS. The author proposed that this is due to the intact compensate mechanism of the mitochondria, which allowed mitochondrial fusion to happen, thus fulfilling the energic need of cells (Bartsakoulia et al., 2018). Besides, an E3 ubiquitin ligase membrane-associated ring- $\mathrm{CH}$ finger protein 5 (MARCH5) could degrade MiD49, promoting mitochondrial fusion as a stress-response mechanism (Xu et al., 2016). MiD49 and MiD51 regulate cytochrome $\mathrm{C}$ release and then activate the Bax/Bak pathway. Loss of MiD49 and MiD51 prevents cristae from remodeling during apoptosis (Otera et al., 2016). Those pieces of evidence indicate that MiD49 and MiD51 are upstream players in mitochondrial fission, acting as the first-line responders upon apoptotic stress.

\section{Optic Atrophy 1}

OPA1 is a mitochondrial dynamin-like GTPase that localizes to the mitochondrial inner membrane. OPA1 comprises 32 exons and spans more than $40 \mathrm{~kb}$. The deduced protein from OPA1 contained 978 amino acids, with a size of about $120 \mathrm{kDa}$. OPA1 binds membranes enriched in negatively charged phospholipids, such as cardiolipin, and promotes membrane tubulation. OPA1 itself has a basal rate of GTP hydrolysis, which enhances its association with negatively charged phospholipids. OPA1 transfers into highly ordered oligomers when associated with lipids. OPA1 assembles on the lipid tubule surface, forming a protein-membrane structure similar to those of typical dynamins. Those shreds of evidence proved that OPA1 could stimulate higher-order membrane assembly, promote GTP hydrolysis, and transform membranes into tubules (Ban et al., 2010). OPA1 is also a pivotal player during apoptosis, and it is essential in remodeling cristae and releasing cytochrome C. Oligomerization of OPA1 regulates apoptosis by maintaining the tension of cristae connections (Frezza et al., 2006). Intrinsic apoptotic signals cause dissociation of OPA1 oligomers and release cytochrome $\mathrm{C}$ as the caspase activator into the intermembrane space. This process is also crucial in maintaining the mtDNA genome (Amati-Bonneau et al., 2008; Elachouri et al., 2011). OPA1 has ten isoforms. The isoform of OPA1 containing exon $4 \mathrm{~b}$ could anchor the mitochondrial nucleoids to the inner membrane, whereas isoforms containing exon 4 are essential in upholding the membrane potential (Olichon et al., 2007; Elachouri et al., 2011). The long isoform of OPA1 (L-OPA1) is responsible for locking the intermembrane space inside cristae. After disassembly of L-OPA1-containing complexes, cytochrome $\mathrm{c}$ release leads to mitochondrial fragmentation (Jiang et al., 2014). OPA1 interacts with OMA1 zinc metallopeptidase (OMA1) and Mitochondrial-Escape 1-like 1 (YME1L1) to function correctly and maintain the L-OPA1 status.

Conversely, knockout of both Omal and Ymell in mouse cardiomyocytes prevents the conversion of L-Opal to S-Opal forms and restores the standard mitochondrial architecture, in addition to protecting Ymell mutant mice from cardiomyopathy and early death. Functional mitochondrial fusion mediated by L-OPA1 preserves cardiac function, and mitochondrial fragmentation may trigger dilated cardiomyopathy and heart failure (Wai et al., 2015). Fresh new research found that knockout of the phosphatidyl glycerophosphate synthase PSG1 causes cardiolipin reduction, thus rescuing mitochondrial fragmentation caused by OPA1 dysfunction (Cretin et al., 2021). OPAl is another major player in maintaining the mitochondrial architecture, regulating apoptosis, and sustaining the homeostasis of mitochondrial fusion and fission.

\section{Mitofusin 1 and Mitofusin 2}

MFN1 (Mitofusin 1) and MFN2 (Mitofusin 2) were initially discovered as the human homolog of the Drosophila and yeast protein fuzzy onion (Fzo), which regulate mitochondrial fusion. Fzo is crucial for spermatogenesis. Expression of Fzo is tightly restricted to the male germline, and it promotes the mRNA accumulation in both sperm cells and spermatids (Hermann et al., 1998; Hwa et al., 2002). The crystal structure of MFN1 showed that it has a GTPase domain and a C-terminal tail. A three-helix bundle that extends from the GTPase was determined, along with another extending from the C-terminal domain, together forming a classical configuration of the bacterial dynamin-like protein. MFN1 forms a dimer when GTP is attached and plays a role in the clustering of vesicles, including the membrane-anchoring GTP-binding domain, which requires undisrupted GTP hydrolysis. As a result, MFN1 tethers mitochondrial outer membranes via a nucleotide-dependent dimerization (Qi et al., 2016). Under anaerobic conditions, mitochondrial elongation is mainly regulated by SIRT1-mediated MFN1 deacetylation, while MFN2 induces mitochondrial fusion by facilitating ER-mitochondrial contact sites (Basso et al., 2018).

MFN2 was first designated as KIAA0214, containing 19 exons; the protein encoded by MFN2 comprised 757 amino acids, containing an ATP/GTP-binding motif. Similarly, MFN2 has a GTPase domain and a C-terminal tail, sharing approximately $60 \%$ of the identity of MFN1. MFN2 targets mitochondria with a predicted bipartite transmembrane domain (Santel and Fuller, 2001). Knockdown of Mfn2 in rat myotubes reduced glucose oxidation by $30 \%$. Inhibition of Mfn2 also leads to diminished mitochondrial membrane potential and aerobic cellular respiration (Bach et al., 2003). The authors further found that MFN2 was repressed under obesity. MFN2 expression was 39\% lower in obese rats and $43 \%$ lower in obese humans than in lean controls (Bach et al., 2003). Obesity-induced MFN2 repression was also associated with the decline of mitochondrial ETC complexes I, II, III, and V (Pich et al., 2005). The promoters of apoptosis, BAX, and BAK play roles in mitochondrial fusion and regulate MFN2. BAX activates mitochondrial fusion by assembling MFN2, redirecting to the submitochondrial area, and alternating its GTP-binding state (Karbowski et al., 2006). Overexpression of $M f n 2$ initiates apoptosis in rat vascular smooth muscle cells and prevents neointima formation after angioplasty. Attenuation of Mfn2 protects vascular muscle cells from damage by ROS (Guo et al., 2007). The authors further found that the proapoptotic effect MFN2 encaptured was mediated by the Akt 
signaling pathway (Guo et al., 2007). The location where MFN2 was enriched was then found to be the ER-mitochondrial interface. Interfering Mfn2 in Hela cells disrupted ER morphology and loosened mitochondrial-ER junctions, reducing calcium uptake upon stimuli (de Brito and Scorrano, 2008). Experiments in human $T$ lymphocytes further proved this hypothesis. MFN2 increased buffering of intracellular $\mathrm{Ca}^{2+}$ (Luchsinger et al., 2016). MFN2 has to be precisely modified to achieve its normal function as well. Phosphorylated MFN2 was found as a parkin receptor in eliminating impaired mitochondria, indicating the quality controller role of MFN2 (Chen and Dorn, 2013). The molecular analysis determined that MFN2 achieves normal function via the rightful peptide-peptide interaction. The PINK1 kinase-mediated phosphorylation of MFN2 through ser378 is adjacent to the determinant of MFN2 activity interactions of met376 and his380 with $\mathrm{Asp}^{725}$ and $\mathrm{Leu}^{727}$ (Rocha et al., 2018). Mouse $M f n 2$ mutant lacking the Pink1 phosphorylation sites inhibited mitochondrial Parkin translocation, repressing mitophagy (Gong et al., 2015). Furthermore, manipulating mitofusin conformations by an engineered cell-permeant mini peptide can reverse mitochondrial abnormalities in human fibroblasts and neurons (Franco et al., 2016). Overall, mitofusins are the central player in maintaining mitochondrial fusion and fission equilibrium, thus keeping mitochondria in a dynamic homeostasis to achieve normal cellular respiratory function.

\section{Solute Carrier Family $\mathbf{2 5}$ Member $\mathbf{4 6}$}

SLC25A46 was first found to be one of the fourteen solute carrier proteins in the central nervous system (Haitina et al., 2006). The protein encoded by SLC25A46 comprising 418 amino acids was the homolog of yeast Ugol in humans. Ugol is a mitochondrial solute in the outer membrane that acts as a fusion factor. SLC25A46 was integrated inside the outer mitochondrial membrane (Abrams et al., 2015). There is a hypothesis that SLC25A46 acts as a transporter across the outer membrane or as a protein adaptor resembling Ugol (Vásquez-Trincado et al., 2016). However, human SLC25A46 failed to rescue ugol deletion in S. cerevisiae. Mouse Slc25a46 protein has an approximately 100-residue N-terminal domain, followed by three tandem repeats of similar size. Each contains two transmembrane domains separated by a large loop, along with a distinct Px $(\mathrm{D} / \mathrm{E}) \mathrm{xx}(\mathrm{R} / \mathrm{K})$ motif. The exact function of SLC25A46 is yet to be explored. SLC25A46 interacts with MFN2 and OPA1 and may combine with the cristae-restructuring protein MIC60 (Mitofilin) beside the ER-mitochondrial contact site. Besides, abrupted cristae have been determined in multiple disease models carrying the Slc25a46 mutation (Abrams et al., 2015; Duchesne et al., 2017; Li et al., 2017). Janer and colleagues found SLC25A46 loss of function secondarily altered ER morphology, leading to premature cellular senescence. SLC25A46 also coordinated with the ER membrane protein complex EMC and altered phospholipid composition within mitochondria. The authors proposed that SLC25A46 plays a role in a mitochondrial-ER interface and facilitates lipid transfer; dysfunction of SLC25A46 altered mitochondrial dynamics, finally leading to cell death (Janer et al., 2016). The deletion of mouse and zebrafish
Slc25a46 leads to premature death, severe mitochondrial dysfunction, and hyperfused mitochondria (Abrams et al., 2015; Li et al., 2017). However, whether the hyperfusion of mitochondria in Slc25a46 mutants is primary or secondary is still elucidated.

\section{HUMAN NEUROLOGICAL DISEASES CAUSED BY DYNAMIC DYSFUNCTION OF MITOCHONDRIA}

Changes in mitochondrial function and morphology are constantly studied in humans as disease triggers, and abnormal mitochondrial dynamics have been implicated as an early event in the pathogenesis of many diseases. Cardiac, metabolic, kidney, and neurological disorders can be linked to dynamic dysfunction of mitochondria. However, mitochondrial dysfunction of fission and fusion are mostly secondary under those conditions. Herein, we summarized disorders caused by mutation of mitochondrial dynamic-regulating genes primarily (Table 1). The most reported disorders caused by mitochondrial dynamic-controlled gene mutations are OPA1-related autosomal dominant atrophy and MFN2-related Charco-Marie-Tooth diseases (CMT). Nevertheless, DRP1, INF2, MIEF1, MIEF2, and SLC25A46 play essential roles, especially in neurological disorders.

\section{Charcot-Marie-Tooth Diseases 2A}

CMT2A occurs due to heterozygous mutation of MFN2, featured as sensory and motor neuropathy of peripheral nerves (Züchner et al., 2004). Unlike CMT, CMT2A is an axonopathy with no or slight reduction of neuronal conduction velocity (VásquezTrincado et al., 2016). The typical clinical features include hammertoes, foot drop, distal limb muscle weakness and atrophy, hyporeflexia, or areflexia. MFN1 and MFN2 interact with Miro and Milton proteins that form the molecular complex linking MFN2 to kinesin motors. Mutation in MFN2 affects this interaction, leading to impairment in axonal mitochondrial transport (Adebayo et al., 2021). Early onset is often associated with more severe cases, resembling dominant optic atrophy caused by OPA1 mutations (Hamedani et al., 2021). Besides, deletion of MFN2 is perinatally lethal to the embryo in both murine and canine models, likely due to lack of protein stability.

\section{Dominant Optic Atrophy}

OPA1-related DOAs commonly diagnosed in early childhood are characterized by progressive bilateral visual loss, color vision loss, visual field defects, optic nerve atrophy, and optic disc excavation (Kjer et al., 1996). DOA plus is associated with central or peripheral neuronal defects featured by deafness, ataxia, myopathy, and progressive external ophthalmoplegia (AmatiBonneau et al., 2005; Amati-Bonneau et al., 2008; Yu-Wai-Man et al., 2010). Pathological studies suggested retinal ganglion cell degeneration and myelin loss of the optic nerve (Alexander et al., 2000; Kim et al., 2005). There have been reports of variable phenotypes and incomplete penetrance. DOA is the most 
TABLE 1 | Primary disorders caused by mutation of mitochondrial dynamic-regulating genes.

\begin{tabular}{|c|c|c|c|c|}
\hline Genes & Phenotype & Inheritance & References & $\underset{\text { ID }}{\text { Omim }}$ \\
\hline \multirow[t]{4}{*}{ DRP1 } & $\begin{array}{l}\text { Encephalopathy, lethal, due to defective mitochondrial } \\
\text { peroxisomal fission } 1\end{array}$ & $A D, A R$ & Waterham et al. (2007); Yoon et al. (2016) & 614,388 \\
\hline & Optic atrophy 5 & $A D$ & Gerber et al. (2017) & 610,708 \\
\hline & Refractory epilepsy & $A D$ & Vanstone et al. (2016) & $\mathrm{N} / \mathrm{A}$ \\
\hline & Mitochondrial cardiac encephalopathy & $A D$ & Vandeleur et al. (2019) & $\mathrm{N} / \mathrm{A}$ \\
\hline \multirow[t]{2}{*}{ INF2 } & Focal segmental glomerulosclerosis & $A D$ & Brown et al. (2010) & 613,237 \\
\hline & Charcot-Marie-Tooth disease, dominant intermediate E & $A D$ & Boyer et al. (2011) & 614,455 \\
\hline MIEF1 & Singular late-onset optic neuropathy & $A D$ & Charif et al. (2021) & N/A \\
\hline MIEF2 & Combined oxidative phosphorylation deficiency 49 & AR & Bartsakoulia et al. (2018) & 619,024 \\
\hline \multirow[t]{5}{*}{ OPA1 } & Autosomal dominant optic atrophy & $A D$ & Alexander et al. (2000); Delettre et al. (2000); Pesch et al. (2001) & 165,500 \\
\hline & Optic atrophy and sensorineural deafness & $A D$ & Amati-Bonneau et al. (2005); Li et al. (2005) & 125,250 \\
\hline & Behr syndrome & $\mathrm{AR}$ & Schaaf et al. (2011); Bonneau et al. (2014) & 210,000 \\
\hline & Mitochondrial DNA depletion syndrome 14 & $\mathrm{AR}$ & Spiegel et al. (2016) & 616,896 \\
\hline & Normal-tension glaucoma & $\mathrm{AR}$ & Aung et al. (2002) & 606,657 \\
\hline \multirow[t]{2}{*}{ MFN2 } & Charcot-Marie-Tooth neuropathy type 2A & $A D, A R$ & $\begin{array}{l}\text { Züchner et al. (2004); Kijima et al. (2005); Guillet et al. (2010); } \\
\text { Polke et al. (2011) }\end{array}$ & 609,260 \\
\hline & Hereditary motor and sensory neuropathy with optic atrophy & $A D$ & Züchner et al. (2006) & 601,152 \\
\hline \multirow[t]{3}{*}{ SLC25A46 } & Hereditary motor and sensory neuropathy, type VIB & AR & Abrams et al. (2015) & 616,505 \\
\hline & $\begin{array}{l}\text { Progressive myoclonic ataxia with optic atrophy and } \\
\text { neuropathy }\end{array}$ & $\mathrm{AR}$ & Charlesworth et al. (2016) & N/A \\
\hline & Pontocerebellar hypoplasia, type $1 \mathrm{E}$ & $\mathrm{AR}$ & Wan et al. (2016); van Dijk et al. (2017); Braunisch et al. (2018) & 619,303 \\
\hline
\end{tabular}

reported inherited optic atrophy with a 1/12,000-1/50,000 prevalence (Kjer et al., 1996). Most deleterious OPA1 mutations are likely due to haploinsufficiency, resulting in dominant-negative effects (Stuppia et al., 2015). DOA plus can show mitochondrial deletion in some muscle fibers with OXPHOS dysfunction following OPA1 loss of function (Chen et al., 2010). Consistent with their protein function, unsurprisingly, DOA plus and CMT2A associated with MFN2 share many features; they both affected motor and sensory neurons. Due to the relatively long axons, these neurons are considered sensitive to mitochondrial dynamics dysfunction.

\section{Encephalopathy Due to Mitochondrial and Peroxisomal Fission 1}

Patients affected by DRP1 mutations were initially reported to be suffering from lethal encephalopathy due to mitochondrial and peroxisomal fission 1. Symptoms of this desperate disorder include microcephaly, abnormal brain development, refractory epilepsy, optic atrophy, persistent lactic acidemia, and elevated plasma very long-chain fatty acids (VLCFA) (Fahrner et al., 2016; Sheffer et al., 2016). Abnormal gyral patterns, bilateral cerebral volume loss, demyelination, thinning of the corpus callosum, and T2-weighted hyperintense lesions in the cortex were visible on MRI in a certain proportion of the patients (Waterham et al., 2007). The number of peroxisomes of the patient's cells was reduced while the sizes varied significantly. The severity of this disorder varies, but most of the patients die in early childhood. Fluorescence microscopy revealed elongated mitochondria concentrated around the nucleus (Waterham et al., 2007). Mitochondrial respiratory chain enzyme activity showed decreased complex IV activity and reduced ATP production (Sheffer et al., 2016). Cardiac involvement is also frequently observed in patients with DRP1 mutations. Dilated left ventricle, reduced ejection fraction, and reduced shortening fraction were visible on the echocardiogram. Post-Morten electronic microscopy found filamented mitochondria inside the patient cardiomyocytes.

\section{Alzheimer's Disease}

Amyloid protein $\beta$ accumulates in the brains of patients with $\mathrm{AD}$ (Huang and Mucke, 2012). Disruption of dynamic mitochondrial homeostasis could be a crucial factor of neuronal apoptosis, thus leading to abnormal neurodevelopment. Nitric oxide (NO) was thought to be involved in the process of Amyloid protein $\beta$ production. It is an essential regulatory factor that regulates mitochondrial division through S-nitrosylated drp1 (SNODRP1), reducing synapses and damaging neurons. It regulates mitochondrial division, which reduces synapses and damages neurons. However, cysteine mutation can prevent DRP1 nitrosylation and eliminate its neurotoxicity. Furthermore, nitrosylation can eliminate its neurotoxicity. In addition, SNODRP1 is also highly expressed in the brains of patients with AD (Cho et al., 2009). Researchers found that primary neurons of the $\mathrm{Tau}^{-1-}$ mouse transfected with truncated Tau showed fragmented mitochondria. They further found that a significant reduction of OPA1 accompanied mitochondrial fragmentation. They concluded that the Tau could impair mitochondrial dynamics by reducing OPA1 levels, leading to mitochondrial impairment in AD (Pérez et al., 2018).

\section{Parkinson's Disease}

Disruption of mitochondrial activity may be associated with PD, especially under the dysfunction of PINK1 and Parkin. PINK1 and Parkin are localized in the mitochondria (Wang et al., 2021). PINK1 protects against mitochondrial dysfunction under stress 
by phosphorylating mitochondrial proteins. PINK1 is also involved in eliminating damaged mitochondria via mitophagy by mediating activation and translocation of Parkin; it targets Parkin to dysfunctional mitochondria with low membrane potential through the phosphorylation of MFN2 (Chen and Dorn, 2013). As an E3 ubiquitin ligase, Parkin could induce the ubiquitination of MFN1, MFN2, FIS1, and DRP1; fragmented mitochondria was initiated once the functions of PINK1 and Parkin are damaged (Ziviani et al., 2010; Wang et al., 2011). Thus, PD pathology is somehow related to mitochondrial dynamics; however, it tends to be secondary rather than the primary cause.

\section{Huntington's Disease}

Over-fragmented mitochondria were identified in patients with HD and animal models, leading to a decreased OXPHOS rate (Bossy-Wetzel et al., 2008). In HD, mitochondrial abnormalities, morphological changes, and dysfunction are visible. Hungtitin protein (HTT), located in mitochondria, might be the cause of mitochondrial fragmentation. HTT mutants could accumulate in the body and trigger DRP1 dysfunction, leading to mitochondrial transport abnormalities and ultimately leading to neuronal apoptosis (Song et al., 2011; Shirendeb et al., 2012). Of note, long CAG repeats in HTT could promote the age-dependent expansion of pathogenic mtDNA heteroplasmy in HD lymphoblasts (Wang et al., 2021). Thus, they concluded that mtDNA quality is declining along with the HD's process, indicating a role of HTT in mtDNA quality control. Controversially, the mitochondrial membrane was found to impact the HTT aggregation as well (Adegbuyiro et al., 2021). Collectively, the mitochondrial dynamic is heavily involved in $\mathrm{HD}$; however, it is mainly subordinate.

\section{EMERGING THERAPEUTIC APPROACHES TARGETING PRIMARY MITOCHONDRIAL FISSION-FUSION ABNORMALITIES}

Previous studies that aimed to intervene in mitochondrial fusion and fission worked either by overexpression or silencing the dysfunctional genes. Promising results from laboratories came out with pharmaceutical potential. DRP1 has to translocate to mitochondria to ensure fragmentation, which requires rightful posttranslational phosphorylation. PKA, Cam kinase, and Pim1 mediate phosphorylation in Drp1 Ser ${ }^{637}$. Phosphorylated rat Pim 1 increases Drp1Ser ${ }^{637}$ phosphorylation and inhibits Drp1 localization to the mitochondria, protecting rat cardiomyocytes from P53 upregulated modulator apoptosis (PUMA) (Din et al., 2013). A couple of years ago, Franco and colleagues reported that a mini-peptide derived from MFN2, capable of competing with endogenous peptide-peptide interactions hampering MFN1 and MFN2 into inactive conformations, transformed the latter into a more active construction, thus promoting mitochondrial fusion (Franco et al., 2016). However, this kind of peptide might still be costly to manufacture, hampering the clinical therapeutic use. Rocha and coworkers used in silico screening to identify a mitofusin agonist. They returned axonal mitochondrial traffic to normal in the sciatic nerves of MFN2 mutant mice and mitigated the dysmotility and fragmentation depolarization and clattering of mitochondria (Rocha et al., 2018). Most recently, Franco and collaborators reported a small molecule named MiM111, which can activate mutant-inhibited MFN2 and normalize neuromuscular function in CMT2A, further reversing axon and myocyte atrophy. They claimed that MiM111 was the first preclinical candidate treatment for CMT2A (Franco et al., 2020). Furthermore, as a widely used tool for gene therapy, adeno-associated virus (AAV)-mediated gene replacement or editing is now undergoing clinical translation. Using AAVSlc25a46, Yang and colleagues ameliorated SLC25A46-related mitochondrial hyperfusion in a murine model and recovered the movement disorder and sciatic nerve demyelination, extending the longevity of the Slc25a46 ${ }^{-1-}$ mouse (Yang et al., 2020).

On the other hand, inhibitors of mitochondrial fission may hold promise as therapeutic targets to treat patients with mitochondrial over-fission, providing a protective effect for mitochondria viability under certain circumstances, thus attracting researcher's interest. Mdivi (Cassidy-Stone et al., 2008), P110 (Qi et al., 2013), and Dynasore (Macia et al., 2006) are mitochondrial fission inhibitors. Cassidy and colleagues found that Mdivi inhibited the assembly of Drp1 and its GTPase enzymatic activity in vitro by binding the outside of the GTPase domain, thus inhibiting GTPase activity. However, Mdivi treatment does not impact Drp1 expression (Xie et al., 2013). Moreover, multiple studies investigated the inhibitor function of Mdivi in vitro and proved its protecting role of mitochondrial function under apoptotic stress (Tang et al., 2013; Wappler et al., 2013). Furthermore, Mdivi could rescue both mitochondrial overfission and improve mitochondrial function in the CRND8 AD mouse model (Wang et al., 2017) and multiple mouse models of cerebral ischemia (Flippo et al., 2018) or organ injury (Tábara et al., 2014; Rogers et al., 2021). Qi and colleagues developed a Drp1 inhibitor named P110 and found that P110 decreases Fis1 expression and reduces excessive mitochondrial fission in cultured neurons. Furthermore, P110 reduced ROS production, improving mitochondrial membrane potential and mitochondrial integrity (Qi et al., 2013). Marcia and coworkers identified Dynasore from 16,000 small molecules as a fission inhibitor. They found that Dynasore interferes with the GTPase activity of Dynamin1, Dynamin 2, and Drp1 (Macia et al., 2006). Gao et al. further investigated the protective effects of Dynasore against ischemia/reperfusion injury in mice. Dynasore increased cardiomyocyte survival and reduced the depletion of cellular ATP (Gao et al., 2013).

To date, there is no clinical trial registered or any human case reported yet using Mdivi-1. The main reason is that the mitochondrial over-fission mouse model is often lethal to the embryo (Chen et al., 2003; Davies et al., 2007). Therefore, researchers could not test the therapeutic and side effects on animal models, not to mention trials on humans. Later on, mouse models capable of surviving after birth carrying the MFN2 point mutation were built (Detmer et al., 2008; Cartoni et al., 2010). However, until recently, no report had tested the therapeutic effect of the mitochondrial fission inhibitors on these models. 
More therapeutic explorations are needed to determine their protecting efficacy from mutant protein-induced neuronal damage and promising candidates to treat patients.

\section{MITOCHONDRIAL DYNAMICS AND REPRODUCTION}

Female reproductive mitochondria are associated with oocytes and embryos because they donate ATP via OXPHOS (MayPanloup et al., 2016). The physiological processes include spindle assembly, chromosome separation, oocyte maturation, fertilization, and embryonic development (Rodríguez-Varela and Labarta, 2020). However, mitochondrial function in female germ cells appears to be more than superficial.

Mitochondrial replication is constantly in progress during oogenesis, and the number of mitochondria increases as oocytes mature (Jansen and de Boer, 1998). The mtDNA copy number maintains a relatively stable state in the mature oocyte and early embryo in many mammals, including humans (Pikó and Taylor, 1987; Ebert et al., 1988; Kameyama et al., 2007; Hashimoto et al., 2017). Therefore, mitochondrial replication will be suspended during this time, resulting in relatively steady levels of mitochondria (Jansen and de Boer, 1998; Collado-Fernandez et al., 2012). Moreover, before the blastocyst stage, the mature oocyte and embryonic metabolism primarily depend on pyruvate by OXPHOS (Collado-Fernandez et al., 2012; Bradley and Swann, 2019). Consequently, the number and quality of mitochondria in oocytes and embryos must be sufficient to provide enough energy for embryonic development. These mitochondria must be relatively evenly distributed to blastomeres until mitochondrial biogenesis resumes in blastomeres (May-Panloup et al., 2021). Thus, mitochondria quantitative and morphological abnormalities in the oocyte and early embryo, or the defective distribution of mitochondria in blastomeres, will lead to reduced OXPHOS, resulting in fertilization failure and embryonic development dysfunction (Van Blerkom et al., 1995; Van Blerkom et al., 2000).

In contrast to somatic cells, oocytes and early embryos have radically different mitochondrial morphology and submitochondrial structure. The cytoplasm comprises many spherical mitochondria with parallel or vaulted cristae and pale matrices in the early stages of development. These mitochondria gather with other organelles around the nucleus to form the Balbiani's vitelline body. In mature oocytes, mitochondria are minor and present with a round or oval shape with arched cristae. Most mitochondria form unique structures with the tubular membrane of the smooth endoplasmic reticulum (M-SER aggregates) and vesicles (MV complexes) (Szöllösi et al., 1986; Motta et al., 1988). These structures are supposed to reserve material and membranes for subsequent fertilization and embryo development (Motta et al., 2000). Mitochondrial morphology does not change significantly in the zygote and 2-cell embryo after fertilization (round or oval mitochondria that are $0.4-0.6 \mu \mathrm{m}$ in length). Longer mitochondria $(1.5-2.5 \mu \mathrm{m})$ and more abundant cristae are observed by transmission electron microscopy in 4-cell embryos, indicating increased mitochondrial activity (Motta et al., 2000). The mitochondria in 6- to 8-cell embryos become much more prolonged $(2.5 \mu \mathrm{m})$, and mitochondrial cristae are more abundant (Sundström et al., 1981; Motta et al., 2000). Recovery of mtDNA replication first occurs in trophoblastic cells of the blastocyst, which coincides with a significant increase in embryo energy demand (Houghton, 2006; St. John et al., 2010; St. John, 2014).

From here, we see that mitochondria maintain a spherical or elliptic shape in the mature oocyte and the early embryo, with a maximum length of $2.5 \mathrm{um}$, and sparse, immature cristae. The typical elongated or rod-shaped mitochondria with abundant transverse cristae were not observed in the oocyte or embryo. Consequently, mitochondrial activity is low, and oxygen consumption and ATP production are also reduced due to the undifferentiated mitochondrial morphology (Van Blerkom et al., 1995). Therefore, the mitochondria's total number and function, especially the mitochondria with high membrane potential (usually reflecting high mitochondrial activity), are essential for embryonic development (Au et al., 2005). In addition, the spatial location of mitochondria also seems to be related to the embryo's developmental competence. Mitochondria must be distributed to cytoplasmic locations with high energy requirements to support critical events in oocyte maturation and embryonic development, such as pronounced mitochondrial aggregation (Van Blerkom et al., 2000). Mitochondria maintaining the low active forms (round mitochondria with sparse cristae) in oocytes and embryos most of the time is beneficial to reducing ROS production as much as possible (Ramalho-Santos et al., 2009) and thus minimizes oxidative damage to the oocytes and embryos. The mitochondria change the morphology in response to the increased demand for ATP only when a particular event occurs (cell division) (Van Blerkom, 2011). Therefore, either mitochondrial dysfunction or excessive function in oocytes and embryos will result in embryonic development impairment.

The role of mitochondrial dynamic-related proteins in germ cells and embryos has been studied in some knockout mice and specific knockout mouse germ cells and embryos. The specific deletion of Drp1 in oocytes leads to aggregation of malformed fusion mitochondria, impaired calcium oscillation, secretory function, meiosis of oocytes, and female mice infertility caused by oocyte maturation and ovulation disorders in an agedependent way (Udagawa et al., 2014). This infertility could correlate with human embryo fragments on the third day (Otasevic et al., 2016). In addition, the use of Mdivi-1, a DRP1 inhibitor, reduced the formation of pig blastocysts with decreased mitochondrial membrane potential and increased ROS (Yeon et al., 2015). Mfn1/Mfn2 double knockout oocytes showed mitochondrial structural damage, including reduced mitochondrial cristae and decreased matrix density, resulting in the arrest of oocyte development and impaired oocyte-granulosa cell interaction (Zhang et al., 2019a). Mfn2 knockout mouse oocytes displayed decreased oocyte maturation and fertilization rates and an alteration in mitochondrial distribution and spindle morphology (Liu et al., 2016), which further suggested the role of mitochondrial dynamics in 


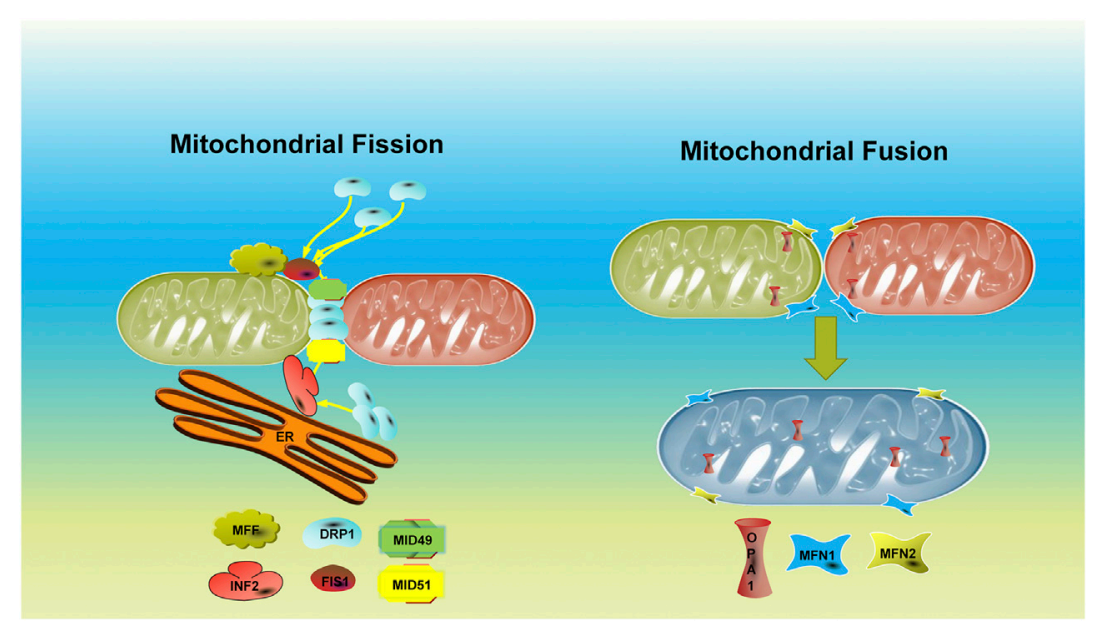

FIGURE 1 | Schemes and fundamental regulators of mitochondrial dynamics.

regulating chromosome separation in oocytes. In addition, siRNA-induced reduction of $M f n 2$ expression diminished the rate of blastocyst formation in mice and the number of embryos passing through the third cell division (Zhao et al., 2015). Mitochondrial fusion may be required to support this milestone event as activating the embryonic genome. Mfn2 deletion in oocytes causes female infertility resulting from mitochondrial dysfunction, oocyte maturation, and follicular development blocking (Zhang et al., 2019b). Embryos that survived $M f n 2$ knockout showed decreased levels of ATP, mitochondrial membrane potential, and mtDNA deficiency and increased levels of mitochondrial apoptosis (Zhao et al., 2015), further suggesting that mitochondrial fusion is essential for maintaining oocyte and embryonic development. In contrast, the irregular mitochondrial distributions, including mitochondrial aggregation and ER coaggregation and increased contact between mitochondria and mitochondria and mitochondria and the ER, were detectable in overexpression of $M f n 1$ or $M f n 2$ oocytes. The unusual behavior of mitochondria and the ER results in impaired calcium homeostasis and abnormal chromosomal segregation during meiosis (Wakai et al., 2014). Consequently, similarly, deficiency or overexpression of mitochondrial dynamic-related proteins will develop from mitochondrial morphological malformations, unusually low or high mitochondrial activity, increased ROS production, and disturbance of intracellular signal molecules.

Therefore, mitochondrial fission and fusion homeostasis are of great consequence to the maintenance of oocyte development, maturation, and embryo development. How can we improve the oocyte and embryo development ability by modulating mitochondrial dynamics, especially improving the reproduction ability of patients with oocyte maturation disorders or embryo development arrest? When in vitro culture conditions are altered, mitochondrial morphology, distribution, and function may be affected (Barnett et al., 1997; Squirrell et al., 2001). The addition of melatonin in the medium of in vitro maturation has been shown to promote the maturation of immature oocytes and subsequent embryonic development by increasing the production of ATP, reducing intracellular ROS generation, and lowering calcium levels (Zou et al., 2020).

According to research, adding proper levels of melatonin to the culture medium resulted in the best oocyte maturation, fertilization, and blastocyst formation rates. Too low or too high of a melatonin concentration could not achieve the best effects ( $\mathrm{Li}$ et al., 2019). Mitochondrial fission and fusion homeostasis is the critical factor in ensuring the moderate mitochondrial activity in oocytes and embryos that can maintain the normal development of embryos and avoid oxidative damage. As more molecules or drugs are discovered and confirmed, molecules regulating mitochondrial dynamics may be a future target for improving female fertility.

\section{CONCLUSION}

In summary, the homeostasis of mitochondrial dynamics is a multiprotein regulation physiological process (Figure 1). Therefore, abnormalities of relevant genes or proteins in somatic cells or germ cells will lead to disorders of the mitochondrial dynamic balance due to impaired mitochondrial morphology and function, leading to organ dysfunction and diseases. DRP1, MFN1, and MFN2 are closely related to female reproduction. Targeted treatment of the gene or protein may be a therapeutic method for mitochondrial dynamic-related diseases. However, there is still further to go from experimental research to clinical application.

\section{AUTHOR CONTRIBUTIONS}

WZ and LY wrote the manuscript. The other authors revised the manuscript. All authors approved the submitted version. 


\section{FUNDING}

This study was funded by the National Natural Science Foundation of China (82001635 and 82001449) and the Youth Development Programme of the First Affiliated Hospital of Anhui Medical University, 2018 (2849).

\section{REFERENCES}

Abrams, A. J., Hufnagel, R. B., Rebelo, A., Zanna, C., Patel, N., Gonzalez, M. A., et al. (2015). Mutations in SLC25A46, Encoding a UGO1-like Protein, Cause an Optic Atrophy Spectrum Disorder. Nat. Genet. 47, 926-932. doi:10.1038/ ng.3354

Adachi, Y., Itoh, K., Yamada, T., Cerveny, K. L., Suzuki, T. L., Macdonald, P., et al. (2016). Coincident Phosphatidic Acid Interaction Restrains Drp1 in Mitochondrial Division. Mol. Cel 63, 1034-1043. doi:10.1016/ j.molcel.2016.08.013

Adebayo, M., Singh, S., Singh, A. P., and Dasgupta, S. (2021). Mitochondrial Fusion and Fission: The fine-tune Balance for Cellular Homeostasis. Faseb J. 35, e21620. doi:10.1096/fj.202100067r

Adegbuyiro, A., Sedighi, F., Jain, P., Pinti, M. V., Siriwardhana, C., Hollander, J. M., et al. (2021). Mitochondrial Membranes Modify Mutant Huntingtin Aggregation. Biochim. Biophys. Acta Biomembr. 1863, 183663. doi:10.1016/ j.bbamem.2021.183663

Alexander, C., Votruba, M., Pesch, U. E. A., Thiselton, D. L., Mayer, S., Moore, A., et al. (2000). OPA1, Encoding a Dynamin-Related GTPase, Is Mutated in Autosomal Dominant Optic Atrophy Linked to Chromosome 3q28. Nat. Genet. 26, 211-215. doi:10.1038/79944

Amati-Bonneau, P., Guichet, A., Olichon, A., Chevrollier, A., Viala, F., Miot, S., et al. (2005). OPA1 R445H Mutation in Optic Atrophy Associated with Sensorineural Deafness. Ann. Neurol. 58, 958-963. doi:10.1002/ana.20681

Amati-Bonneau, P., Valentino, M. L., Reynier, P., Gallardo, M. E., Bornstein, B., Boissière, A., et al. (2008). OPA1 Mutations Induce Mitochondrial DNA Instability and Optic Atrophy 'plus' Phenotypes. Brain 131, 338-351. doi:10.1093/brain/awm298

Au, H.-K., Yeh, T.-S., Kao, S.-H., Tzeng, C.-R., and Hsieh, R.-H. (2005). Abnormal Mitochondrial Structure in Human Unfertilized Oocytes and Arrested Embryos. Ann. N.Y Acad. Sci. 1042, 177-185. doi:10.1196/annals.1338.020

Aung, T., Ocaka, L., Ebenezer, N. D., Morris, A. G., Krawczak, M., Thiselton, D. L., et al. (2002). A Major Marker for normal Tension Glaucoma: Association with Polymorphisms in the OPAl Gene. Hum. Genet. 110, 52-56. doi:10.1007/ s00439-001-0645-7

Bach, D., Pich, S., Soriano, F. X., Vega, N., Baumgartner, B., Oriola, J., et al. (2003). Mitofusin-2 Determines Mitochondrial Network Architecture and Mitochondrial Metabolism. J. Biol. Chem. 278, 17190-17197. doi:10.1074/ jbc.m212754200

Ban, T., Heymann, J. A. W., Song, Z., Hinshaw, J. E., and Chan, D. C. (2010). OPA1 Disease Alleles Causing Dominant Optic Atrophy Have Defects in CardiolipinStimulated GTP Hydrolysis and Membrane Tubulation. Hum. Mol. Genet. 19, 2113-2122. doi:10.1093/hmg/ddq088

Barnett, D. K., Clayton, M. K., Kimura, J., and Bavister, B. D. (1997). Glucose and Phosphate Toxicity in Hamster Preimplantation Embryos Involves Disruption of Cellular Organization, Including Distribution of Active Mitochondria. Mol. Reprod. Dev. 48, 227-237. doi:10.1002/(sici)1098-2795(199710)48:2<227:aid$\operatorname{mrd} 10>3.0 . \mathrm{co} ; 2-\mathrm{v}$

Bartsakoulia, M., Pyle, A., Troncoso-Chandía, D., Vial-Brizzi, J., Paz-Fiblas, M. V., Duff, J., et al. (2018). A Novel Mechanism Causing Imbalance of Mitochondrial Fusion and Fission in Human Myopathies. Hum. Mol. Genet. 27, 1186-1195. doi: $10.1093 / \mathrm{hmg} / \mathrm{ddy} 033$

Basso, V., Marchesan, E., Peggion, C., Chakraborty, J., von Stockum, S., Giacomello, M., et al. (2018). Regulation of ER-Mitochondria Contacts by Parkin via Mfn2. Pharmacol. Res. 138, 43-56. doi:10.1016/j.phrs.2018.09.006

Benard, G., Bellance, N., James, D., Parrone, P., Fernandez, H., Letellier, T., et al. (2007). Mitochondrial Bioenergetics and Structural Network Organization. J. Cel Sci. 120, 838-848. doi:10.1242/jcs.03381

\section{ACKNOWLEDGMENTS}

We acknowledge members of the NHC Key Laboratory of Study on Abnormal Gametes and Reproductive Tract (Anhui Medical University) for constructive comments and discussions.

Bindschadler, M., and McGrath, J. L. (2004). Formin' New Ideas about Actin Filament Generation. Proc. Natl. Acad. Sci. 101, 14685-14686. doi:10.1073/ pnas.0406317101

Bonneau, D., Colin, E., Oca, F., Ferré, M., Chevrollier, A., Guéguen, N., et al. (2014). Early-onset Behr Syndrome Due to Compound Heterozygous Mutations in OPA1. Brain A J. Neurol. 137, e301. doi:10.1093/brain/awu184

Bossy-Wetzel, E., Petrilli, A., and Knott, A. B. (2008). Mutant Huntingtin and Mitochondrial Dysfunction. Trends Neurosci. 31, 609-616. doi:10.1016/ j.tins.2008.09.004

Boyer, O., Nevo, F., Plaisier, E., Funalot, B., Gribouval, O., Benoit, G., et al. (2011). INF2Mutations in Charcot-Marie-Tooth Disease with Glomerulopathy. N. Engl. J. Med. 365, 2377-2388. doi:10.1056/nejmoa1109122

Bradley, J., and Swann, K. (2019). Mitochondria and Lipid Metabolism in Mammalian Oocytes and Early Embryos. Int. J. Dev. Biol. 63, 93-103. doi:10.1387/ijdb.180355ks

Braunisch, M. C., Gallwitz, H., Abicht, A., Diebold, I., Holinski-Feder, E., Van Maldergem, L., et al. (2018). Extension of the Phenotype of Biallelic Loss-OfFunction Mutations in SLC25A46 to the Severe Form of Pontocerebellar Hypoplasia Type I. Clin. Genet. 93, 255-265. doi:10.1111/cge.13084

Breckenridge, D. G., Kang, B.-H., Kokel, D., Mitani, S., Staehelin, L. A., and Xue, D. (2008). Caenorhabditis elegans Drp-1 and Fis-2 Regulate Distinct Cell-Death Execution Pathways Downstream of Ced-3 and Independent of Ced-9. Mol. Cel 31, 586-597. doi:10.1016/j.molcel.2008.07.015

Brown, E. J., Schlöndorff, J. S., Becker, D. J., Tsukaguchi, H., Tonna, S. J., Uscinski, A. L., et al. (2010). Mutations in the Formin Gene INF2 Cause Focal Segmental Glomerulosclerosis. Nat. Genet. 42, 72-76. doi:10.1038/ng.505

Cai, Q., and Tammineni, P. (2016). Alterations in Mitochondrial Quality Control in Alzheimer's Disease. Front Cel Neurosci. 10, 24. doi:10.3389/ fncel.2016.00024

Cartoni, R., Arnaud, E., Médard, J.-J., Poirot, O., Courvoisier, D. S., Chrast, R., et al. (2010). Expression of Mitofusin 2R94Q in a Transgenic Mouse Leads to Charcot-Marie-Tooth Neuropathy Type 2A. Brain A J. Neurol. 133, 1460-1469. doi:10.1093/brain/awq082

Cassidy-Stone, A., Chipuk, J. E., Ingerman, E., Song, C., Yoo, C., Kuwana, T., et al (2008). Chemical Inhibition of the Mitochondrial Division Dynamin Reveals its Role in Bax/Bak-dependent Mitochondrial Outer Membrane Permeabilization. Develop. Cel 14, 193-204. doi:10.1016/j.devcel.2007.11.019

Chan, D. C. (2012). Fusion and Fission: Interlinked Processes Critical for Mitochondrial Health. Annu. Rev. Genet. 46, 265-287. doi:10.1146/annurevgenet-110410-132529

Charif, M., Wong, Y. C., Kim, S., Guichet, A., Vignal, C., Zanlonghi, X., et al. (2021). Dominant Mutations in MIEF1 Affect Mitochondrial Dynamics and Cause a Singular Late Onset Optic Neuropathy. Mol. Neurodegener. 16, 12. doi:10.1186/s13024-021-00431-w

Charlesworth, G., Balint, B., Mencacci, N. E., Carr, L., Wood, N. W., and Bhatia, K. P. (2016). SLC25A46 Mutations Underlie Progressive Myoclonic Ataxia with Optic Atrophy and Neuropathy. Mov Disord. 31, 1249-1251. doi:10.1002/ mds. 26716

Chen, H., Detmer, S. A., Ewald, A. J., Griffin, E. E., Fraser, S. E., and Chan, D. C. (2003). Mitofusins Mfn1 and Mfn2 Coordinately Regulate Mitochondrial Fusion and Are Essential for Embryonic Development. J. Cel Biol. 160, 189-200. doi:10.1083/jcb.200211046

Chen, H., Vermulst, M., Wang, Y. E., Chomyn, A., Prolla, T. A., McCaffery, J. M., et al. (2010). Mitochondrial Fusion Is Required for mtDNA Stability in Skeletal Muscle and Tolerance of mtDNA Mutations. Cell 141, 280-289. doi:10.1016/ j.cell.2010.02.026

Chen, Y., and Dorn, G. W. (2013). PINK1-Phosphorylated Mitofusin 2 Is a Parkin Receptor for Culling Damaged Mitochondria. Science 340, 471-475. doi:10.1126/science. 1231031 
Chhabra, E. S., and Higgs, H. N. (2006). INF2 Is a WASP Homology 2 MotifContaining Formin that Severs Actin Filaments and Accelerates Both Polymerization and Depolymerization. J. Biol. Chem. 281, 26754-26767. doi:10.1074/jbc.m604666200

Cho, D.-H., Nakamura, T., Fang, J., Cieplak, P., Godzik, A., Gu, Z., et al. (2009). S-Nitrosylation of Drp1 Mediates $\beta$-Amyloid-Related Mitochondrial Fission and Neuronal Injury. Science 324, 102-105. doi:10.1126/science.1171091

Clark-Walker, G. D., and Miklos, G. L. (1975). Complementation in Cytoplasmic Petite Mutants of Yeast to Form Respiratory Competent Cells. Proc. Natl. Acad. Sci. 72, 372-375. doi:10.1073/pnas.72.1.372

Collado-Fernandez, E., Picton, H. M., and Dumollard, R. (2012). Metabolism throughout Follicle and Oocyte Development in Mammals. Int. J. Dev. Biol. 56, 799-808. doi:10.1387/ijdb.120140ec

Cretin, E., Lopes, P., Vimont, E., Tatsuta, T., Langer, T., Gazi, A., et al. (2021). High-throughput Screening Identifies Suppressors of Mitochondrial Fragmentation in OPA1 Fibroblasts. EMBO Mol. Med. 13, e13579. doi:10.15252/emmm.202013579

Davies, V. J., Hollins, A. J., Piechota, M. J., Yip, W., Davies, J. R., White, K. E., et al. (2007). Opal Deficiency in a Mouse Model of Autosomal Dominant Optic Atrophy Impairs Mitochondrial Morphology, Optic Nerve Structure and Visual Function. Hum. Mol. Genet. 16, 1307-1318. doi:10.1093/hmg/ddm079

de Brito, O. M., and Scorrano, L. (2008). Mitofusin 2 Tethers Endoplasmic Reticulum to Mitochondria. Nature 456, 605-610. doi:10.1038/nature07534

Delettre, C., Lenaers, G., Griffoin, J.-M., Gigarel, N., Lorenzo, C., Belenguer, P., et al. (2000). Nuclear Gene OPA1, Encoding a Mitochondrial Dynamin-Related Protein, Is Mutated in Dominant Optic Atrophy. Nat. Genet. 26, 207-210. doi:10.1038/79936

Detmer, S. A., Velde, C. V., Cleveland, D. W., and Chan, D. C. (2008). Hindlimb Gait Defects Due to Motor Axon Loss and Reduced Distal Muscles in a Transgenic Mouse Model of Charcot-Marie-Tooth Type 2A. Hum. Mol. Genet. 17, 367-375. doi:10.1093/hmg/ddm314

Din, S., Mason, M., Völkers, M., Johnson, B., Cottage, C. T., Wang, Z., et al. (2013). Pim-1 Preserves Mitochondrial Morphology by Inhibiting Dynamin-Related Protein 1 Translocation. Proc. Natl. Acad. Sci. 110, 5969-5974. doi:10.1073/ pnas. 1213294110

Duchesne, A., Vaiman, A., Castille, J., Beauvallet, C., Gaignard, P., Floriot, S., et al. (2017). Bovine and Murine Models Highlight Novel Roles for SLC25A46 in Mitochondrial Dynamics and Metabolism, with Implications for Human and Animal Health. Plos Genet. 13, e1006597. doi:10.1371/journal.pgen.1006597

Dumesic, D. A., Meldrum, D. R., Katz-Jaffe, M. G., Krisher, R. L., and Schoolcraft, W. B. (2015). Oocyte Environment: Follicular Fluid and Cumulus Cells Are Critical for Oocyte Health. Fertil. Sterility 103, 303-316. doi:10.1016/ j.fertnstert.2014.11.015

Ebert, K. M., Liem, H., and Hecht, N. B. (1988). Mitochondrial DNA in the Mouse Preimplantation Embryo. Reproduction 82, 145-149. doi:10.1530/jrf.0.0820145

Elachouri, G., Vidoni, S., Zanna, C., Pattyn, A., Boukhaddaoui, H., Gaget, K., et al. (2011). OPA1 Links Human Mitochondrial Genome Maintenance to mtDNA Replication and Distribution. Genome Res. 21, 12-20. doi:10.1101/ gr.108696.110

Fahrner, J. A., Liu, R., Perry, M. S., Klein, J., and Chan, D. C. (2016). A Novel De Novo Dominant Negative Mutation inDNM1Limpairs Mitochondrial Fission and Presents as Childhood Epileptic Encephalopathy. Am. J. Med. Genet. 170, 2002-2011. doi:10.1002/ajmg.a.37721

Flippo, K. H., Gnanasekaran, A., Perkins, G. A., Ajmal, A., Merrill, R. A., Dickey, A. S., et al. (2018). AKAP1 Protects from Cerebral Ischemic Stroke by Inhibiting Drp1-Dependent Mitochondrial Fission. J. Neurosci. 38, 8233-8242. doi:10.1523/jneurosci.0649-18.2018

Franco, A., Dang, X., Walton, E. K., Ho, J. N., Zablocka, B., Ly, C., et al. (2020). Burst Mitofusin Activation Reverses Neuromuscular Dysfunction in Murine CMT2A. Elife 9, e61119. doi:10.7554/eLife.61119

Franco, A., Kitsis, R. N., Fleischer, J. A., Gavathiotis, E., Kornfeld, O. S., Gong, G., et al. (2016). Correcting Mitochondrial Fusion by Manipulating Mitofusin Conformations. Nature 540, 74-79. doi:10.1038/nature20156

Frederick, R. L., and Shaw, J. M. (2007). Moving Mitochondria: Establishing Distribution of an Essential Organelle. Traffic 8, 1668-1675. doi:10.1111/ j.1600-0854.2007.00644.x

Frezza, C., Cipolat, S., Martins de Brito, O., Micaroni, M., Beznoussenko, G. V., Rudka, T., et al. (2006). OPA1 Controls Apoptotic Cristae Remodeling
Independently from Mitochondrial Fusion. Cell 126, 177-189. doi:10.1016/ j.cell.2006.06.025

Frieden, M., James, D., Castelbou, C., Danckaert, A., Martinou, J.-C., and Demaurex, N. (2004). Ca2+ Homeostasis during Mitochondrial Fragmentation and Perinuclear Clustering Induced by hFis1. J. Biol. Chem. 279, 22704-22714. doi:10.1074/jbc.m312366200

Gandre-Babbe, S., and van der Bliek, A. M. (2008). The Novel Tail-Anchored Membrane Protein Mff Controls Mitochondrial and Peroxisomal Fission in Mammalian Cells. MBoC 19, 2402-2412. doi:10.1091/mbc.e07-12-1287

Gao, D., Zhang, L., Dhillon, R., Hong, T.-T., Shaw, R. M., and Zhu, J. (2013). Dynasore Protects Mitochondria and Improves Cardiac Lusitropy in Langendorff Perfused Mouse Heart. PloS one 8, e60967. doi:10.1371/ journal.pone.0060967

Gardner, D. K., and Harvey, A. J. (2015). Blastocyst Metabolism. Reprod. Fertil. Dev. 27, 638-654. doi:10.1071/rd14421

Gerber, S., Charif, M., Chevrollier, A., Chaumette, T., Angebault, C., Kane, M. S., et al. (2017). Mutations in DNM1L, as in OPA1, Result in Dominant Optic Atrophy Despite Opposite Effects on Mitochondrial Fusion and Fission. Brain A J. Neurol. 140, 2586-2596. doi:10.1093/brain/awx219

Gong, G., Song, M., Csordas, G., Kelly, D. P., Matkovich, S. J., and Dorn, G. W. (2015). Parkin-mediated Mitophagy Directs Perinatal Cardiac Metabolic Maturation in Mice. Science 350, aad2459. doi:10.1126/science.aad2459

Guillet, V., Gueguen, N., Verny, C., Ferre, M., Homedan, C., Loiseau, D., et al. (2010). Adenine Nucleotide Translocase Is Involved in a Mitochondrial Coupling Defect in MFN2-Related Charcot-Marie-Tooth Type 2A Disease. Neurogenetics 11, 127-133. doi:10.1007/s10048-009-0207-z

Guo, X., Chen, K.-H., Guo, Y., Liao, H., Tang, J., and Xiao, R.-P. (2007). Mitofusin 2 Triggers Vascular Smooth Muscle Cell Apoptosis via Mitochondrial Death Pathway. Circ. Res. 101, 1113-1122. doi:10.1161/circresaha.107.157644

Gurel, P. S., Mu, A., Guo, B., Shu, R., Mierke, D. F., and Higgs, H. N. (2015). Assembly and Turnover of Short Actin Filaments by the Formin INF2 and Profilin. J. Biol. Chem. 290, 22494-22506. doi:10.1074/jbc.m115.670166

Haitina, T., Lindblom, J., Renström, T., and Fredriksson, R. (2006). Fourteen Novel Human Members of Mitochondrial Solute Carrier Family 25 (SLC25) Widely Expressed in the central Nervous System. Genomics 88, 779-790. doi:10.1016/ j.ygeno.2006.06.016

Hamedani, A. G., Wilson, J. A., Avery, R. A., and Scherer, S. S. (2021). Optic Neuropathy in Charcot-Marie-Tooth Disease. J. Neuroophthalmol. 41, 233-238. doi:10.1097/wno.0000000000000965

Harvey, A. J. (2019). Mitochondria in Early Development: Linking the Microenvironment, Metabolism and the Epigenome. Reproduction 157, R159-R179. doi:10.1530/rep-18-0431

Hashimoto, S., Morimoto, N., Yamanaka, M., Matsumoto, H., Yamochi, T., Goto, H., et al. (2017). Quantitative and Qualitative Changes of Mitochondria in Human Preimplantation Embryos. J. Assist. Reprod. Genet. 34, 573-580. doi:10.1007/s10815-017-0886-6

Hermann, G. J., Thatcher, J. W., Mills, J. P., Hales, K. G., Fuller, M. T., Nunnari, J., et al. (1998). Mitochondrial Fusion in Yeast Requires the Transmembrane GTPase Fzolp. J. Cel Biol. 143, 359-373. doi:10.1083/jcb.143.2.359

Houghton, F. D. (2006). Energy Metabolism of the Inner Cell Mass and Trophectoderm of the Mouse Blastocyst. Differentiation 74, 11-18. doi:10.1111/j.1432-0436.2006.00052.x

Huang, Y., and Mucke, L. (2012). Alzheimer Mechanisms and Therapeutic Strategies. Cell 148, 1204-1222. doi:10.1016/j.cell.2012.02.040

Hwa, J. J., Hiller, M. A., Fuller, M. T., and Santel, A. (2002). Differential Expression of the Drosophila Mitofusin Genes Fuzzy Onions (Fzo) and Dmfn. Mech. Develop. 116, 213-216. doi:10.1016/s0925-4773(02)00141-7

Ihenacho, U. K., Meacham, K. A., Harwig, M. C., Widlansky, M. E., and Hill, R. B. (2021). Mitochondrial Fission Protein 1: Emerging Roles in Organellar Form and Function in Health and Disease. Front. Endocrinol. 12, 660095. doi:10.3389/fendo.2021.660095

Iwasawa, R., Mahul-Mellier, A.-L., Datler, C., Pazarentzos, E., and Grimm, S. (2011). Fis1 and Bap31 Bridge the Mitochondria-ER Interface to Establish a Platform for Apoptosis Induction. EMBO J. 30, 556-568. doi:10.1038/ emboj.2010.346

James, D. I., Parone, P. A., Mattenberger, Y., and Martinou, J.-C. (2003). hFis1, A Novel Component of the Mammalian Mitochondrial Fission Machinery. J. Biol. Chem. 278, 36373-36379. doi:10.1074/jbc.m303758200 
Janer, A., Prudent, J., Paupe, V., Fahiminiya, S., Majewski, J., Sgarioto, N., et al. (2016). SLC 25A46 Is Required for Mitochondrial Lipid Homeostasis and Cristae Maintenance and Is Responsible for Leigh Syndrome. EMBO Mol. Med. 8, 1019-1038. doi:10.15252/emmm.201506159

Jansen, R. P. S., and de Boer, K. (1998). The Bottleneck: Mitochondrial Imperatives in Oogenesis and Ovarian Follicular Fate. Mol. Cell Endocrinol. 145, 81-88. doi:10.1016/s0303-7207(98)00173-7

Jendrach, M., Mai, S., Pohl, S., Vöth, M., and Bereiter-Hahn, J. (2008). Short- and Long-Term Alterations of Mitochondrial Morphology, Dynamics and mtDNA after Transient Oxidative Stress. Mitochondrion 8, 293-304. doi:10.1016/ j.mito.2008.06.001

Jiang, X., Jiang, H., Shen, Z., and Wang, X. (2014). Activation of Mitochondrial Protease OMA1 by Bax and Bak Promotes Cytochrome C Release during Apoptosis. Proc. Natl. Acad. Sci. 111, 14782-14787. doi:10.1073/ pnas. 1417253111

Kalia, R., Wang, R. Y.-R., Yusuf, A., Thomas, P. V., Agard, D. A., Shaw, J. M., et al. (2018). Structural Basis of Mitochondrial Receptor Binding and Constriction by DRP1. Nature 558, 401-405. doi:10.1038/s41586-018-0211-2

Kamerkar, S. C., Kraus, F., Sharpe, A. J., Pucadyil, T. J., and Ryan, M. T. (2018). Dynamin-related Protein 1 Has Membrane Constricting and Severing Abilities Sufficient for Mitochondrial and Peroxisomal Fission. Nat. Commun. 9, 5239. doi:10.1038/s41467-018-07543-w

Kameyama, Y., Filion, F., Yoo, J. G., and Smith, L. C. (2007). Characterization of Mitochondrial Replication and Transcription Control during Rat Early Development In Vivo and In Vitro. Reproduction 133, 423-432. doi:10.1530/ rep-06-0263

Karbowski, M., Norris, K. L., Cleland, M. M., Jeong, S.-Y., and Youle, R. J. (2006). Role of Bax and Bak in Mitochondrial Morphogenesis. Nature 443, 658-662. doi:10.1038/nature05111

Kijima, K., Numakura, C., Izumino, H., Umetsu, K., Nezu, A., Shiiki, T., et al. (2005). Mitochondrial GTPase Mitofusin 2 Mutation in Charcot?Marie?Tooth Neuropathy Type 2A. Hum. Genet. 116, 23-27. doi:10.1007/s00439-004-1199-2

Kim, J. Y., Hwang, J.-M., Ko, H. S., Seong, M.-W., Park, B.-J., and Park, S. S. (2005). Mitochondrial DNA Content Is Decreased in Autosomal Dominant Optic Atrophy. Neurology 64, 966-972. doi:10.1212/01.wnl.0000157282.76715.b1

Kjer, B., Eiberg, H., Kjer, P., and Rosenberg, T. (1996). Dominant Optic Atrophy Mapped to Chromosome 3q Region. II. Clinical and Epidemiological Aspects. Acta Ophthalmol. Scand. 74, 3-7. doi:10.1111/j.1600-0420.1996.tb00672.x

Kleele, T., Rey, T., Winter, J., Zaganelli, S., Mahecic, D., Perreten Lambert, H., et al. (2021). Distinct Fission Signatures Predict Mitochondrial Degradation or Biogenesis. Nature 593, 435-439. doi:10.1038/s41586-021-03510-6

Kobayashi, S., Tanaka, A., and Fujiki, Y. (2007). Fis1, DLP1, and Pex11p Coordinately Regulate Peroxisome Morphogenesis. Exp. Cel Res. 313, 1675-1686. doi:10.1016/j.yexcr.2007.02.028

Korobova, F., Ramabhadran, V., and Higgs, H. N. (2013). An Actin-dependent Step in Mitochondrial Fission Mediated by the ER-Associated Formin INF2. Science 339, 464-467. doi:10.1126/science. 1228360

Li, C., Kosmorsky, G., Zhang, K., Katz, B. J., Ge, J., and Traboulsi, E. I. (2005). Optic Atrophy and Sensorineural Hearing Loss in a Family Caused by an R445HOPA1 Mutation. Am. J. Med. Genet. 138a, 208-211. doi:10.1002/ ajmg.a.30794

Li, Y., Liu, H., Wu, K., Liu, H., Huang, T., Chen, Z. J., et al. (2019). Melatonin Promotes Human Oocyte Maturation and Early Embryo Development by Enhancing Clathrin-Mediated Endocytosis. J. Pineal Res. 67, e12601. doi:10.1111/jpi.12601

Li, Z., Peng, Y., Hufnagel, R. B., Hu, Y.-C., Zhao, C., Queme, L. F., et al. (2017). Loss of SLC25A46 Causes Neurodegeneration by Affecting Mitochondrial Dynamics and Energy Production in Mice. Hum. Mol. Genet. 26, 3776-3791. doi:10.1093/ $\mathrm{hmg} / \mathrm{ddx} 262$

Liesa, M., and Shirihai, O. S. (2013). Mitochondrial Dynamics in the Regulation of Nutrient Utilization and Energy Expenditure. Cel Metab. 17, 491-506. doi:10.1016/j.cmet.2013.03.002

Liu, Q., Kang, L., Wang, L., Zhang, L., and Xiang, W. (2016). Mitofusin 2 Regulates the Oocytes Development and Quality by Modulating Meiosis and Mitochondrial Function. Sci. Rep. 6, 30561. doi:10.1038/srep30561

Losón, O. C., Liu, R., Rome, M. E., Meng, S., Kaiser, J. T., Shan, S.-o., et al. (2014). The Mitochondrial Fission Receptor MiD51 Requires ADP as a Cofactor. Structure 22, 367-377. doi:10.1016/j.str.2014.01.001
Losón, O. C., Meng, S., Ngo, H., Liu, R., Kaiser, J. T., and Chan, D. C. (2015). Crystal Structure and Functional Analysis of MiD49, a Receptor for the Mitochondrial Fission Protein Drp1. Protein Sci. 24, 386-394. doi:10.1002/ pro. 2629

Losón, O. C., Song, Z., Chen, H., and Chan, D. C. (2013). Fis1, Mff, MiD49, and MiD51 Mediate Drp1 Recruitment in Mitochondrial Fission. MBoC 24, 659-667. doi:10.1091/mbc.e12-10-0721

Luchsinger, L. L., de Almeida, M. J., Corrigan, D. J., Mumau, M., and Snoeck, H.W. (2016). Mitofusin 2 Maintains Haematopoietic Stem Cells with Extensive Lymphoid Potential. Nature 529, 528-531. doi:10.1038/nature16500

Macia, E., Ehrlich, M., Massol, R., Boucrot, E., Brunner, C., and Kirchhausen, T. (2006). Dynasore, a Cell-Permeable Inhibitor of Dynamin. Develop. Cel 10, 839-850. doi:10.1016/j.devcel.2006.04.002

Malena, A., Loro, E., Di Re, M., Holt, I. J., and Vergani, L. (2009). Inhibition of Mitochondrial Fission Favours Mutant over Wild-type Mitochondrial DNA. Hum. Mol. Genet. 18, 3407-3416. doi:10.1093/hmg/ddp281

Manor, U., Bartholomew, S., Golani, G., Christenson, E., Kozlov, M., Higgs, H., et al. (2015). A Mitochondria-Anchored Isoform of the Actin-Nucleating Spire Protein Regulates Mitochondrial Division. Elife 4, e08828. doi:10.7554/ eLife. 08828

May-Panloup, P., Boguenet, M., Hachem, H. E., Bouet, P. E., and Reynier, P. (2021). Embryo and its Mitochondria. Antioxidants 10, 139. doi:10.3390/ antiox10020139

May-Panloup, P., Boucret, L., Chao de la Barca, J.-M., Desquiret-Dumas, V., FerréL'Hotellier, V., Morinière, C., et al. (2016). Ovarian Ageing: the Role of Mitochondria in Oocytes and Follicles. Hum. Reprod. Update 22, 725-743. doi:10.1093/humupd/dmw028

Motta, P. M., Nottola, S. A., Makabe, S., and Heyn, R. (2000). Mitochondrial Morphology in Human Fetal and Adult Female Germ Cells. Hum. Reprod. 15 (Suppl. 2), 129-147. doi:10.1093/humrep/15.suppl_2.129

Motta, P. M., Nottola, S. A., Micara, G., and Familiari, G. (1988). Ultrastructure of Human Unfertilized Oocytes and Polyspermic Embryos in an IVF-ET Program. Ann. NY Acad. Sci. 541, 367-383. doi:10.1111/j.17496632.1988.tb22274.x

Olichon, A., Elachouri, G., Baricault, L., Delettre, C., Belenguer, P., and Lenaers, G. (2007). OPA1 Alternate Splicing Uncouples an Evolutionary Conserved Function in Mitochondrial Fusion from a Vertebrate Restricted Function in Apoptosis. Cell Death Differ. 14, 682-692. doi:10.1038/sj.cdd.4402048

Osellame, L. D., Singh, A. P., Stroud, D. A., Palmer, C. S., Stojanovski, D., Ramachandran, R., et al. (2016). Cooperative and Independent Roles of the Drp1 Adaptors Mff, MiD49 and MiD51 in Mitochondrial Fission. J. Cel Sci. 129, 2170-2181. doi: $10.1242 /$ jcs. 185165

Otasevic, V., Surlan, L., Vucetic, M., Tulic, I., Buzadzic, B., Stancic, A., et al. (2016). Expression Patterns of Mitochondrial OXPHOS Components, Mitofusin 1 and Dynamin-Related Protein 1 Are Associated with Human Embryo Fragmentation. Reprod. Fertil. Dev. 28, 319-327. doi:10.1071/rd13415

Otera, H., and Mihara, K. (2011). Discovery of the Membrane Receptor for Mitochondrial Fission GTPase Drp1. Small GTPases 2, 167-172. doi:10.4161/sgtp.2.3.16486

Otera, H., Miyata, N., Kuge, O., and Mihara, K. (2016). Drp1-dependent Mitochondrial Fission via MiD49/51 Is Essential for Apoptotic Cristae Remodeling. J. Cel Biol. 212, 531-544. doi:10.1083/jcb.201508099

Otera, H., Wang, C., Cleland, M. M., Setoguchi, K., Yokota, S., Youle, R. J., et al. (2010). Mff Is an Essential Factor for Mitochondrial Recruitment of Drp1 during Mitochondrial Fission in Mammalian Cells. J. Cel. Biol. 191, 1141-1158. doi:10.1083/jcb.201007152

Palmer, C. S., Elgass, K. D., Parton, R. G., Osellame, L. D., Stojanovski, D., and Ryan, M. T. (2013). Adaptor Proteins MiD49 and MiD51 Can Act Independently of Mff and Fis1 in Drp1 Recruitment and Are Specific for Mitochondrial Fission. J. Biol. Chem. 288, 27584-27593. doi:10.1074/ jbc.m113.479873

Palmer, C. S., Osellame, L. D., Laine, D., Koutsopoulos, O. S., Frazier, A. E., and Ryan, M. T. (2011). MiD49 and MiD51, New Components of the Mitochondrial Fission Machinery. EMBO Rep. 12, 565-573. doi:10.1038/embor.2011.54

Pérez, M. J., Vergara-Pulgar, K., Jara, C., Cabezas-Opazo, F., and Quintanilla, R. A. (2018). Caspase-Cleaved Tau Impairs Mitochondrial Dynamics in Alzheimer's Disease. Mol. Neurobiol. 55, 1004-1018. doi:10.1007/s12035017-0385-x 
Pesch, U. E. A., Leo-Kottler, B., Mayer, S., Jurklies, B., Kellner, U., Apfelstedt-Sylla, E., et al. (2001). OPA1 Mutations in Patients with Autosomal Dominant Optic Atrophy and Evidence for Semi-dominant Inheritance. Hum. Mol. Genet. 10, 1359-1368. doi:10.1093/hmg/10.13.1359

Pich, S., Bach, D., Briones, P., Liesa, M., Camps, M., Testar, X., et al. (2005). The Charcot-Marie-Tooth Type 2A Gene Product, Mfn2, Up-Regulates Fuel Oxidation through Expression of OXPHOS System. Hum. Mol. Genet. 14, 1405-1415. doi:10.1093/hmg/ddi149

Pikó, L., and Taylor, K. D. (1987). Amounts of Mitochondrial DNA and Abundance of Some Mitochondrial Gene Transcripts in Early Mouse Embryos. Develop. Biol. 123, 364-374. doi:10.1016/0012-1606(87)90395-2

Polke, J. M., Laurá, M., Pareyson, D., Taroni, F., Milani, M., Bergamin, G., et al. (2011). Recessive Axonal Charcot-Marie-Tooth Disease Due to Compound Heterozygous Mitofusin 2 Mutations. Neurology 77, 168-173. doi:10.1212/ wnl.0b013e3182242d4d

Qi, X., Qvit, N., Su, Y. C., and Mochly-Rosen, D. (2013). A Novel Drp1 Inhibitor Diminishes Aberrant Mitochondrial Fission and Neurotoxicity. J. Cel Sci. 126, 789-802. doi:10.1242/jcs.114439

Qi, Y., Yan, L., Yu, C., Guo, X., Zhou, X., Hu, X., et al. (2016). Structures of Human Mitofusin 1 Provide Insight into Mitochondrial Tethering. J. Cel Biol. 215, 621-629. doi:10.1083/jcb.201609019

Ramalho-Santos, J., Varum, S., Amaral, S., Mota, P. C., Sousa, A. P., and Amaral, A. (2009). Mitochondrial Functionality in Reproduction: from Gonads and Gametes to Embryos and Embryonic Stem Cells. Hum. Reprod. Update 15, 553-572. doi:10.1093/humupd/dmp016

Reubold, T. F., Faelber, K., Plattner, N., Posor, Y., Ketel, K., Curth, U., et al. (2015). Crystal Structure of the Dynamin Tetramer. Nature 525, 404-408. doi:10.1038/ nature 14880

Richter, V., Palmer, C. S., Osellame, L. D., Singh, A. P., Elgass, K., Stroud, D. A., et al. (2014). Structural and Functional Analysis of MiD51, a Dynamin Receptor Required for Mitochondrial Fission. J. Cel Biol. 204, 477-486. doi:10.1083/ jcb.201311014

Rocha, A. G., Franco, A., Krezel, A. M., Rumsey, J. M., Alberti, J. M., Knight, W. C., et al. (2018). MFN2 Agonists Reverse Mitochondrial Defects in Preclinical Models of Charcot-Marie-Tooth Disease Type 2A. Science 360, 336-341. doi:10.1126/science.aao1785

Rodríguez-Varela, C., and Labarta, E. (2020). Clinical Application of Antioxidants to Improve Human Oocyte Mitochondrial Function: A Review. Antioxidants 9, 1197. doi:10.3390/antiox9121197

Rogers, M. A., Hutcheson, J. D., Okui, T., Goettsch, C., Singh, S. A., Halu, A., et al. (2021). Dynamin-related Protein 1 Inhibition Reduces Hepatic PCSK9 Secretion. Cardiovasc. Res. 117, 2340-2353. doi:10.1093/cvr/cvab034

Santel, A., and Fuller, M. T. (2001). Control of Mitochondrial Morphology by a Human Mitofusin. J. Cel Sci. 114, 867-874. doi:10.1242/jcs.114.5.867

Sathananthan, A. H., and Trounson, A. O. (2000). Mitochondrial Morphology during Preimplantational Human Embryogenesis. Hum. Reprod. 15 (Suppl. 2), 148-159. doi:10.1093/humrep/15.suppl_2.148

Schaaf, C. P., Blazo, M., Lewis, R. A., Tonini, R. E., Takei, H., Wang, J., et al. (2011). Early-onset Severe Neuromuscular Phenotype Associated with Compound Heterozygosity for OPA1 Mutations. Mol. Genet. Metab. 103, 383-387. doi:10.1016/j.ymgme.2011.04.018

Sheffer, R., Douiev, L., Edvardson, S., Shaag, A., Tamimi, K., Soiferman, D., et al. (2016). Postnatal Microcephaly and Pain Insensitivity Due to a De Novo heterozygousDNM1Lmutation Causing Impaired Mitochondrial Fission and Function. Am. J. Med. Genet. 170, 1603-1607. doi:10.1002/ajmg.a.37624

Shen, Q., Yamano, K., Head, B. P., Kawajiri, S., Cheung, J. T. M., Wang, C., et al. (2014). Mutations in Fis1 Disrupt Orderly Disposal of Defective Mitochondria. MBoC 25, 145-159. doi:10.1091/mbc.e13-09-0525

Shirendeb, U. P., Calkins, M. J., Manczak, M., Anekonda, V., Dufour, B., McBride, J. L., et al. (2012). Mutant Huntingtin's Interaction with Mitochondrial Protein Drp1 Impairs Mitochondrial Biogenesis and Causes Defective Axonal Transport and Synaptic Degeneration in Huntington's Disease. Hum. Mol. Genet. 21, 406-420. doi:10.1093/hmg/ddr475

Smirnova, E., Shurland, D.-L., Ryazantsev, S. N., and van der Bliek, A. M. (1998). A Human Dynamin-Related Protein Controls the Distribution of Mitochondria. J. Cel Biol. 143, 351-358. doi:10.1083/jcb.143.2.351

Song, W., Chen, J., Petrilli, A., Liot, G., Klinglmayr, E., Zhou, Y., et al. (2011). Mutant Huntingtin Binds the Mitochondrial Fission GTPase Dynamin-Related
Protein-1 and Increases its Enzymatic Activity. Nat. Med. 17, 377-382. doi: $10.1038 / \mathrm{nm} .2313$

Spiegel, R., Saada, A., Flannery, P. J., Burté, F., Soiferman, D., Khayat, M., et al. (2016). Fatal Infantile Mitochondrial Encephalomyopathy, Hypertrophic Cardiomyopathy and Optic Atrophy Associated with a homozygousOPAlmutation. J. Med. Genet. 53, 127-131. doi:10.1136/ jmedgenet-2015-103361

Squirrell, J. M., Lane, M., and Bavister, B. D. (2001). Altering Intracellular pH Disrupts Development and Cellular Organization in Preimplantation Hamster Embryos1. Biol. Reprod. 64, 1845-1854. doi:10.1095/biolreprod64.6.1845

Steffen, J., and Koehler, C. M. (2018). ER-mitochondria Contacts: Actin Dynamics at the ER Control Mitochondrial Fission via Calcium Release. J. Cel Biol. 217, 15-17. doi:10.1083/jcb.201711075

Stepanyants, N., Macdonald, P. J., Francy, C. A., Mears, J. A., Qi, X., and Ramachandran, R. (2015). Cardiolipin's Propensity for Phase Transition and its Reorganization by Dynamin-Related Protein 1 Form a Basis for Mitochondrial Membrane Fission. MBoC 26, 3104-3116. doi:10.1091/ mbc.e15-06-0330

St. John, J. C., Facucho-Oliveira, J., Jiang, Y., Kelly, R., and Salah, R. (2010). Mitochondrial DNA Transmission, Replication and Inheritance: a Journey from the Gamete through the Embryo and into Offspring and Embryonic Stem Cells. Hum. Reprod. Update 16, 488-509. doi:10.1093/humupd/dmq002

St. John, J. (2014). The Control of mtDNA Replication during Differentiation and Development. Biochim. Biophys. Acta Gen. Subjects 1840, 1345-1354. doi:10.1016/j.bbagen.2013.10.036

Stuppia, G., Rizzo, F., Riboldi, G., Del Bo, R., Nizzardo, M., Simone, C., et al. (2015). MFN2-related Neuropathies: Clinical Features, Molecular Pathogenesis and Therapeutic Perspectives. J. Neurol. Sci. 356, 7-18. doi:10.1016/ j.jns.2015.05.033

Suen, D.-F., Norris, K. L., and Youle, R. J. (2008). Mitochondrial Dynamics and Apoptosis. Genes Develop. 22, 1577-1590. doi:10.1101/gad.1658508

Sundström, P., Nilsson, O., and Liedholm, P. (1981). Cleavage Rate and Morphology of Early Human Embryos Obtained after Artificial Fertilization and Culture. Acta Obstet. Gynecol. Scand. 60, 109-120. doi:10.1111/ aog.1981.60.issue-5

Szöllösi, D., Mandelbaum, J., Plachot, M., Salat-Baroux, J., and Cohen, J. (1986). Ultrastructure of the Human Preovulatory Oocyte. J. Vitro Fert. Embryo Transf. 3, 232-242. doi:10.1007/BF01132810

Tábara, L. C., Poveda, J., Martin-Cleary, C., Selgas, R., Ortiz, A., and Sanchez-Niño, M. D. (2014). Mitochondria-targeted Therapies for Acute Kidney Injury. Expert Rev. Mol. Med. 16, e13. doi:10.1017/erm.2014.14

Tang, W.-X., Wu, W.-H., Qiu, H.-Y., Bo, H., and Huang, S.-M. (2013). Amelioration of Rhabdomyolysis-Induced Renal Mitochondrial Injury and Apoptosis through Suppression of Drp-1 Translocation. J. Nephrol. 26, 1073-1082. doi:10.5301/jn.5000268

Tondera, D., Grandemange, S., Jourdain, A., Karbowski, M., Mattenberger, Y., Herzig, S., et al. (2009). SLP-2 Is Required for Stress-Induced Mitochondrial Hyperfusion. Embo J. 28, 1589-1600. doi:10.1038/emboj.2009.89

Toyama, E. Q., Herzig, S., Courchet, J., Lewis, T. L., Jr., Losón, O. C., Hellberg, K., et al. (2016). AMP-activated Protein Kinase Mediates Mitochondrial Fission in Response to Energy Stress. Science 351, 275-281. doi:10.1126/science.aab4138

Tresse, E., Riera-Ponsati, L., Jaberi, E., Sew, W. Q. G., Ruscher, K., and IssazadehNavikas, S. (2021). IFN- $\beta$ Rescues Neurodegeneration by Regulating Mitochondrial Fission via STAT5, PGAM5, and Drp1. EMBO J. 40, e106868. doi:10.15252/embj.2020106868

Twig, G., Elorza, A., Molina, A. J. A., Mohamed, H., Wikstrom, J. D., Walzer, G., et al. (2008). Fission and Selective Fusion Govern Mitochondrial Segregation and Elimination by Autophagy. EMBO J. 27, 433-446. doi:10.1038/ sj.emboj.7601963

Udagawa, O., Ishihara, T., Maeda, M., Matsunaga, Y., Tsukamoto, S., Kawano, N., et al. (2014). Mitochondrial Fission Factor Drp1 Maintains Oocyte Quality via Dynamic Rearrangement of Multiple Organelles. Curr. Biol. 24, 2451-2458. doi:10.1016/j.cub.2014.08.060

Van Blerkom, J., Davis, P., and Alexander, S. (2000). Differential Mitochondrial Distribution in Human Pronuclear Embryos Leads to Disproportionate Inheritance between Blastomeres: Relationship to Microtubular Organization, ATP Content and Competence. Hum. Reprod. 15, 2621-2633. doi:10.1093/humrep/15.12.2621 
Van Blerkom, J., Davis, P. W., and Lee, J. (1995). Fertilization and Early Embryolgoy: ATP Content of Human Oocytes and Developmental Potential and Outcome after In-Vitro Fertilization and Embryo Transfer. Hum. Reprod. 10, 415-424. doi:10.1093/oxfordjournals.humrep.a135954

Van Blerkom, J. (2011). Mitochondrial Function in the Human Oocyte and Embryo and Their Role in Developmental Competence. Mitochondrion 11, 797-813. doi:10.1016/j.mito.2010.09.012

van Dijk, T., Rudnik-Schöneborn, S., Senderek, J., Hajmousa, G., Mei, H., Dusl, M., et al. (2017). Pontocerebellar Hypoplasia with Spinal Muscular Atrophy (PCH1): Identification of SLC25A46 Mutations in the Original Dutch PCH1 Family. Brain A J. Neurol. 140, e46. doi:10.1093/brain/awx147

Vandeleur, D., Chen, C. V., Huang, E. J., Connolly, A. J., Sanchez, H., and MoonGrady, A. J. (2019). Novel and Lethal Case of Cardiac Involvement in DNM1L Mitochondrial Encephalopathy. Am. J. Med. Genet. 179, 2486-2489. doi:10.1002/ajmg.a.61371

Vanstone, J. R., Smith, A. M., Smith, A. M., McBride, S., Naas, T., Holcik, M., et al. (2016). DNM1L-related Mitochondrial Fission Defect Presenting as Refractory Epilepsy. Eur. J. Hum. Genet. 24, 1084-1088. doi:10.1038/ejhg.2015.243

Vásquez-Trincado, C., García-Carvajal, I., Pennanen, C., Parra, V., Hill, J. A., Rothermel, B. A., et al. (2016). Mitochondrial Dynamics, Mitophagy and Cardiovascular Disease. J. Physiol. 594, 509-525. doi:10.1113/jp271301

Wai, T., García-Prieto, J., Baker, M. J., Merkwirth, C., Benit, P., Rustin, P., et al. (2015). Imbalanced OPA1 Processing and Mitochondrial Fragmentation Cause Heart Failure in Mice. Science 350, aad0116. doi:10.1126/science.aad0116

Wai, T., and Langer, T. (2016). Mitochondrial Dynamics and Metabolic Regulation. Trends Endocrinol. Metab. 27, 105-117. doi:10.1016/ j.tem.2015.12.001

Wakai, T., Harada, Y., Miyado, K., and Kono, T. (2014). Mitochondrial Dynamics Controlled by Mitofusins Define Organelle Positioning and Movement during Mouse Oocyte Maturation. Mol. Hum. Reprod. 20, 1090-1100. doi:10.1093/ molehr/gau064

Wan, J., Steffen, J., Yourshaw, M., Mamsa, H., Andersen, E., Rudnik-Schöneborn, S., et al. (2016). Loss of Function of SLC25A46 Causes Lethal Congenital Pontocerebellar Hypoplasia. Brain 139, 2877-2890. doi:10.1093/brain/aww212

Wang, H., Song, P., Du, L., Tian, W., Yue, W., Liu, M., et al. (2011). Parkin Ubiquitinates Drp1 for Proteasome-dependent Degradation. J. Biol. Chem. 286, 11649-11658. doi:10.1074/jbc.m110.144238

Wang, W., Yin, J., Ma, X., Zhao, F., Siedlak, S. L., Wang, Z., et al. (2017). Inhibition of Mitochondrial Fragmentation Protects against Alzheimer's Disease in Rodent Model. Hum. Mol. Genet. 26, 4118-4131. doi:10.1093/hmg/ddx299

Wang, Y., Guo, X., Ye, K., Orth, M., and Gu, Z. (2021). Accelerated Expansion of Pathogenic Mitochondrial DNA Heteroplasmies in Huntington's Disease. Proc. Natl. Acad. Sci. U S A 118, e2014610118. doi:10.1073/ pnas.2014610118

Wang, Z., Jiang, H., Chen, S., Du, F., and Wang, X. (2012). The Mitochondrial Phosphatase PGAM5 Functions at the Convergence point of Multiple Necrotic Death Pathways. Cell 148, 228-243. doi:10.1016/j.cell.2011.11.030

Wappler, E. A., Institoris, A., Dutta, S., Katakam, P. V. G., and Busija, D. W. (2013). Mitochondrial Dynamics Associated with Oxygen-Glucose Deprivation in Rat Primary Neuronal Cultures. PloS one 8, e63206. doi:10.1371/ journal.pone.0063206

Waterham, H. R., Koster, J., van Roermund, C. W. T., Mooyer, P. A. W., Wanders, R. J. A., and Leonard, J. V. (2007). A Lethal Defect of Mitochondrial and Peroxisomal Fission. N. Engl. J. Med. 356, 1736-1741. doi:10.1056/ nejmoa064436

Xian, H., Yang, Q., Xiao, L., Shen, H.-M., and Liou, Y.-C. (2019). STX17 Dynamically Regulated by Fis1 Induces Mitophagy via Hierarchical Macroautophagic Mechanism. Nat. Commun. 10, 2059. doi:10.1038/s41467019-10096-1

Xie, N., Wang, C., Lian, Y., Zhang, H., Wu, C., and Zhang, Q. (2013). A Selective Inhibitor of Drp1, Mdivi-1, Protects against Cell Death of Hippocampal Neurons in Pilocarpine-Induced Seizures in Rats. Neurosci. Lett. 545, 64-68. doi:10.1016/j.neulet.2013.04.026

Xu, S., Cherok, E., Das, S., Li, S., Roelofs, B. A., Ge, S. X., et al. (2016). Mitochondrial E3 Ubiquitin Ligase MARCH5 Controls Mitochondrial Fission and Cell Sensitivity to Stress-Induced Apoptosis through Regulation of MiD49 Protein. MBoC 27, 349-359. doi:10.1091/mbc.e15-09-0678
Yang, L., Slone, J., Li, Z., Lou, X., Hu, Y. C., Queme, L. F., et al. (2020). Systemic Administration of AAV-Slc25a46 Mitigates Mitochondrial Neuropathy in Slc25a46-/- Mice. Hum. Mol. Genet. 29, 649. doi:10.1093/hmg/ddz277

Yeon, J.-Y., Min, S.-H., Park, H.-J., Kim, J.-W., Lee, Y.-H., Park, S.-Y., et al. (2015). Mdivi-1, Mitochondrial Fission Inhibitor, Impairs Developmental Competence and Mitochondrial Function of Embryos and Cells in Pigs. J. Reprod. Develop. 61, 81-89. doi:10.1262/jrd.2014-070

Yoon, G., Malam, Z., Paton, T., Marshall, C. R., Hyatt, E., Ivakine, Z., et al. (2016). Lethal Disorder of Mitochondrial Fission Caused by Mutations in DNM1L. J. Pediatr. 171, 313-316. doi:10.1016/j.jpeds.2015.12.060

Yoon, Y., Krueger, E. W., Oswald, B. J., and McNiven, M. A. (2003). The Mitochondrial Protein hFis1 Regulates Mitochondrial Fission in Mammalian Cells through an Interaction with the Dynamin-like Protein DLP1. Mol. Cel Biol. 23, 5409-5420. doi:10.1128/mcb.23.15.5409-5420.2003

Yu-Wai-Man, P., Griffiths, P. G., Gorman, G. S., Lourenco, C. M., Wright, A. F., Auer-Grumbach, M., et al. (2010). Multi-system Neurological Disease Is Common in Patients with OPA1 Mutations. Brain A J. Neurol. 133, 771-786. doi:10.1093/brain/awq007

Zhang, M., Bener, M. B., Jiang, Z., Wang, T., Esencan, E., Scott III, R., Iii, et al. (2019). Mitofusin 1 Is Required for Female Fertility and to Maintain Ovarian Follicular reserve. Cell Death Dis. 10, 560. doi:10.1038/s41419019-1799-3

Zhang, M., Bener, M. B., Jiang, Z., Wang, T., Esencan, E., Scott, R., et al. (2019). Mitofusin 2 Plays a Role in Oocyte and Follicle Development, and Is Required to Maintain Ovarian Follicular reserve during Reproductive Aging. Aging 11, 3919-3938. doi:10.18632/aging.102024

Zhao, J., Liu, T., Jin, S., Wang, X., Qu, M., Uhlén, P., et al. (2011). Human MIEF1 Recruits Drp1 to Mitochondrial Outer Membranes and Promotes Mitochondrial Fusion rather Than Fission. EMBO J. 30, 2762-2778. doi:10.1038/emboj.2011.198

Zhao, N., Zhang, Y., Liu, Q., and Xiang, W. (2015). Mfn2 Affects Embryo Development via Mitochondrial Dysfunction and Apoptosis. PloS one 10, e0125680. doi:10.1371/journal.pone.0125680

Ziviani, E., Tao, R. N., and Whitworth, A. J. (2010). Drosophila Parkin Requires PINK1 for Mitochondrial Translocation and Ubiquitinates Mitofusin. Proc. Natl. Acad. Sci. 107, 5018-5023. doi:10.1073/ pnas. 0913485107

Zou, H., Chen, B., Ding, D., Gao, M., Chen, D., Liu, Y., et al. (2020). Melatonin Promotes the Development of Immature Oocytes from the $\mathrm{COH}$ Cycle into Healthy Offspring by Protecting Mitochondrial Function. J. Pineal Res. 68, e12621. doi:10.1111/jpi.12621

Züchner, S., De Jonghe, P., Jordanova, A., Claeys, K. G., Guergueltcheva, V., Cherninkova, S., et al. (2006). Axonal Neuropathy with Optic Atrophy Is Caused by Mutations in Mitofusin 2. Ann. Neurol. 59, 276-281. doi:10.1002/ ana. 20797

Züchner, S., Mersiyanova, I. V., Muglia, M., Bissar-Tadmouri, N., Rochelle, J., Dadali, E. L., et al. (2004). Mutations in the Mitochondrial GTPase Mitofusin 2 Cause Charcot-Marie-Tooth Neuropathy Type 2A. Nat. Genet. 36, 449-451. doi:10.1038/ng1341

Conflict of Interest: The authors declare that the research was conducted in the absence of any commercial or financial relationships that could be construed as a potential conflict of interest.

Publisher's Note: All claims expressed in this article are solely those of the authors and do not necessarily represent those of their affiliated organizations, or those of the publisher, the editors and the reviewers. Any product that may be evaluated in this article, or claim that may be made by its manufacturer, is not guaranteed or endorsed by the publisher.

Copyright () 2021 Zou, Ji, Zhang, Yang and Cao. This is an open-access article distributed under the terms of the Creative Commons Attribution License (CC BY). The use, distribution or reproduction in other forums is permitted, provided the original author(s) and the copyright owner(s) are credited and that the original publication in this journal is cited, in accordance with accepted academic practice. No use, distribution or reproduction is permitted which does not comply with these terms. 